\title{
QCD corrections to the hadronic production of a heavy quark pair and a $W$-boson including decay correlations
}

\author{
Simon Badger ${ }^{a}$ John M. Campbell ${ }^{b}$ and R.K. Ellis ${ }^{b}$ \\ ${ }^{a}$ Niels Bohr International Academy and Discovery Center, \\ The Niels Bohr Institute, Blegdamsvej 17, DK-2100 Copenhagen, Denmark \\ ${ }^{b}$ Theoretical Physics Department, Fermi National Accelerator Laboratory, \\ P.O. Box 500, Batavia, IL 60510, U.S.A. \\ E-mail: simon.badger@nbi.dk, johnmc@fnal.gov, ellis@fnal.gov
}

ABSTRACT: We perform an analytic calculation of the one-loop amplitude for the $W$ boson mediated process $0 \rightarrow d \bar{u} Q \bar{Q} \bar{\ell} \ell$ retaining the mass for the quark $Q$. The momentum of each of the massive quarks is expressed as the sum of two massless momenta and the corresponding heavy quark spinor is expressed as a sum of two massless spinors. Using a special choice for the heavy quark spinors we obtain analytic expressions for the one-loop amplitudes which are amenable to fast numerical evaluation. The full next-to-leading order (NLO) calculation of hadron + hadron $\rightarrow W(\rightarrow e \nu) b \bar{b}$ with massive $b$-quarks is included in the program MCFM. A comparison is performed with previous published work.

Keywords: NLO Computations, Hadronic Colliders, QCD

ARXIV EPRINT: 1011.6647 


\section{Contents}

1 Introduction 1

2 Treatment of massive spinors $\quad 2$

2.1 Special choice for massive spinors 3

3 Setup 5

3.1 Colour decomposition 5

3.2 Tree level amplitudes 6

3.3 Decomposition of one-loop amplitudes into primitive amplitudes $\quad 7$

$\begin{array}{lll}3.4 & \text { Structure of the calculation } & 7\end{array}$

4 The results for primitive amplitude $A_{6}^{\text {lc }} \quad 9$

4.1 Divergent parts 9

$\begin{array}{ll}4.2 \text { Calculation of the box coefficients } & 9\end{array}$

$\begin{array}{lll}\text { 4.2.1 Calculation of } d_{1|2| 3} & 10\end{array}$

$\begin{array}{ll}\text { 4.2.2 Calculation of } d_{1|2| 34} & 12\end{array}$

$\begin{array}{ll}\text { 4.2.3 Calculation of } d_{1|2| 4} & 13\end{array}$

$\begin{array}{lll}4.3 & \text { Calculation of triangle coefficients } & 15\end{array}$

$\begin{array}{lll}\text { 4.3.1 Forde method for triangle coefficients } & 15\end{array}$

$\begin{array}{ll}\text { 4.4 Calculation of bubble and tadpole coefficients } & 18\end{array}$

$\begin{array}{lll}\text { 4.4.1 } & \text { Bubble integral with one massive propagator } & 19\end{array}$

$\begin{array}{ll}4.5 & \text { Calculation of the rational terms } 20\end{array}$

$5 \quad$ The results for primitive amplitude $A_{6}^{\text {sl }} \quad 22$

6 Results for the primitive amplitudes $A_{6}^{l f}$ and $A_{6}^{h f} \quad 24$

$\begin{array}{lll}7 & \text { Renormalization } & 25\end{array}$

8 Implementation into MCFM $\quad 26$

8.1 Checks on the calculation 26

$\begin{array}{ll}8.2 \text { Phenomenology } & 27\end{array}$

9 Conclusions $\quad 29$

$\begin{array}{ll}\text { A Notation for spinor products } & \mathbf{3 0}\end{array}$

$\begin{array}{ll}\text { B Tree level results } & \mathbf{3 0}\end{array}$

B.1 Results for $A\left(1_{g}, 2_{\bar{Q}}, 3_{Q}, 4_{g}\right) \quad 30$

B.2 Results for $A\left(1_{q}, 2_{\bar{q}}, 3_{g}\right) \quad 32$

B.3 Results for $A\left(1_{q}, 2_{\bar{q}}, 3_{g}, 4_{\bar{l}}, 5_{l}\right) \quad 32$ 


\section{Introduction}

One of the most interesting channels currently under study at the Tevatron and the LHC is the final state containing a $W$-boson and jets, where some or all of the produced jets are tagged as containing a bottom quark. Several interesting partonic processes contribute to this final state, for example,

- $W+H(\rightarrow b \bar{b})$

- $W+Z(\rightarrow b \bar{b})$

- $\bar{b}+t\left(\rightarrow W^{+}+b\right)$

- $\bar{t}+t\left(\rightarrow W^{+}+b\right)$

A background to these processes involving heavy bosons and fermions, is the QCD and electroweak process occurring in the collision of hadrons $H_{1}$ and $H_{2}$,

$$
H_{1}+H_{2} \rightarrow W+b+\bar{b}+X .
$$

An example of a partonic subprocess contributing to this process is shown in figure 1. Next-to-leading corrections to this process were first considered in [1], working in the approximation in which the $b$-quark is considered massless. Since in many analyses the $b$ quark is required to have a minimum $p_{T}$, typically $15 \mathrm{GeV}$ or more, in order to be efficiently tagged, the neglect of the mass of the bottom quark is expected to be a good approximation.

After these initial studies, this same process was considered in refs. [2-5], without making the approximation $m_{b}=0$. These studies, performed without the inclusion of decay products of the $W$ boson effectively confirmed that the $m_{b}=0$ approximation is good to a few percent, with the difference parametrically suppressed as $m_{b}^{2} / p_{T}^{2}$. Thus retaining the mass of the $b$-quark extends the prediction to lower values of $p_{T}$. In addition, it allows us treat the case where the two $b$-quarks end up in the same jet and the case when one $b$-quark is either too soft or too forward to be tagged. These kinematic configurations can be important for the Higgs search at the Tevatron and the LHC $[6,7]$.

The purpose of this paper is to repeat the calculation of ref. [2], i.e. including the effects of a finite $b$-quark mass, but also including the spin correlations present in the $W$ boson decay. The calculation is performed using the spinor helicity formalism, with the calculated amplitudes represented as analytic formulae. Although the formulae are not 


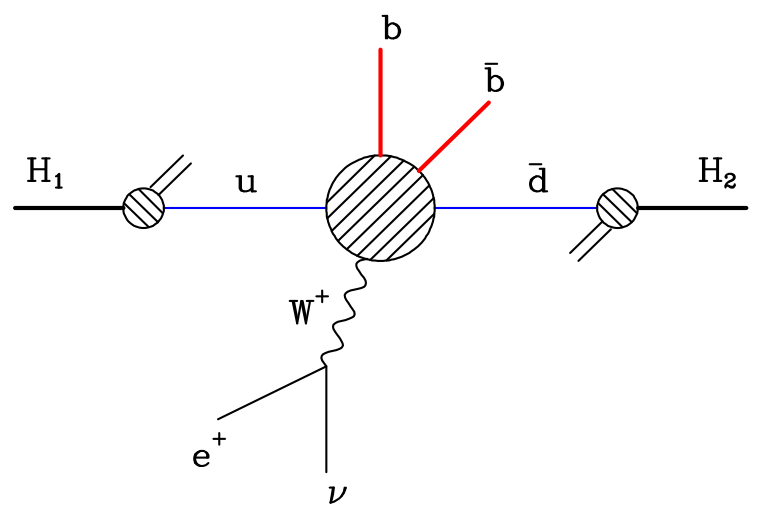

Figure 1. Example of a partonic process contributing to $W b \bar{b}$ production.

compact enough to be presented in their entirety here, they do lead to an efficient and numerically stable code.

Following a four-dimensional unitarity based approach $[8,9]$, we will construct the logarithmic parts of the virtual amplitude using multiple cuts. Developments to this technique utilizing complex momenta [10-13] allow us to compute analytic expressions for the coefficients of the known scalar integrals.

The bulk of our paper is dedicated to a description of the calculation of the one-loop corrections to the process $0 \rightarrow d \bar{u} Q \bar{Q} \bar{\ell} \ell$ retaining the mass for the quark $Q$. The detailed plan of this paper is as follows. In section 2 we present our method for dealing with massive spinors using spinor helicity techniques. Section 3 gives a precise definition of the amplitude that we wish to calculate, including the decomposition into colour stripped amplitudes and the further decomposition into one-loop primitive amplitudes. Sections 4,5 and 6 illustrate our calculation of the leading colour primitive amplitude, $A_{6}^{l c}$, of the sub-leading colour primitive, $A_{6}^{\mathrm{sl}}$, and of the primitive amplitudes containing a closed loop of fermions, $A_{6}^{\mathrm{lf}}$ and $A_{6}^{\mathrm{hf}}$. Section 7 presents the renormalization counterterms. After describing the implementation of the calculation into MCFM in section 8 and comparing with earlier work, we draw some conclusions in section IX.

\section{Treatment of massive spinors}

A method for dealing with a massive particle in the context of the spinor helicity method has been given by Kleiss and Stirling [14]. A massive momentum can always be represented as a sum of two massless momenta. Thus the spinor solution for a massive particle can be expressed in terms of massless spinors by decomposing the physical momentum in terms of the two massless momenta. If we are ultimately going to sum over the spin degrees of freedom, the only constraint that the massive spinors must satisfy is that they should give the standard result for the spin sum after averaging over polarizations, namely,

$$
\begin{aligned}
& \sum_{s= \pm} u_{s}(p, m) \bar{u}_{s}(p, m)=\not p+m \\
& \sum_{s= \pm} v_{s}(p, m) \bar{v}_{s}(p, m)=\not p-m .
\end{aligned}
$$


We can decompose a massive vector into two massless vectors by introducing an arbitrary massless reference vector, $\eta$,

$$
p=p^{b}+\frac{m_{p}^{2}}{\langle\eta|p| \eta]} \eta
$$

In this equation $p^{b}$ is a massless vector. The details of our spinor product notation are given in appendix A. The definitions of massive external spinor wave functions are,

$$
\begin{aligned}
& \bar{u}_{ \pm}\left(p, m ; p^{b}, \eta\right)=\frac{1}{\left\langle\eta \mp \mid p^{b} \pm\right\rangle}\langle\eta \mp|(\not p+m), \\
& v_{ \pm}\left(p, m ; p^{b}, \eta\right)=\frac{1}{\left\langle p^{b} \mp \mid \eta \pm\right\rangle}(\not p-m)|\eta \pm\rangle,
\end{aligned}
$$

where the subscripts \pm label the spin degrees of freedom. In the massless limit these labels correspond to the helicity quantum numbers, but in the massive case they have no such interpretation. Treating the spinors as independent functions of $p^{b}$ and $\eta$ i.e. ignoring the constraint in eq. (2.2) we can show using simple manipulations that,

$$
\begin{aligned}
& \frac{\left[p^{b} \eta\right]}{m} \bar{u}_{-}\left(p, m ; \eta, p^{b}\right)=\bar{u}_{+}\left(p, m ; p^{b}, \eta\right), \\
& \frac{\left\langle p^{b} \eta\right\rangle}{m} v_{+}\left(p, m ; \eta, p^{b}\right)=v_{-}\left(p, m ; p^{b}, \eta\right) .
\end{aligned}
$$

This has the attractive feature that amplitudes with different spin labels can be obtained from one another by exchanging $p^{b}$ and $\eta$. This method has been used in the calculation of one-loop corrections to top production $[15,16]$.

\subsection{Special choice for massive spinors}

In this paper, however, we adopt a different approach. By making a specific choice for the vector $\eta$ in terms of other vectors in the problem we can simplify the calculation of individual amplitudes. In addition, we shall find that for our particular choice of the vector $\eta$, one-loop results for the colour suppressed primitive amplitude can be obtained directly from the corresponding massless amplitude.

The implementation of the Kleiss-Stirling scheme appropriate for the case where we have pairs of massive particles is due to Rodrigo [17]. The two massive momenta $p_{2}$ and $p_{3}$ with equal masses, corresponding to the momenta of the antiquark and quark respectively, are written in terms of lightlike momenta $k_{2}$ and $k_{3}$,

$$
\begin{aligned}
& p_{2}^{\mu}=\frac{1+\beta}{2} k_{2}^{\mu}+\frac{1-\beta}{2} k_{3}^{\mu}, \\
& p_{3}^{\mu}=\frac{1+\beta}{2} k_{3}^{\mu}+\frac{1-\beta}{2} k_{2}^{\mu},
\end{aligned}
$$

where,

$$
\beta=\sqrt{1-4 m^{2} / s_{23}}, \quad \beta_{ \pm}=\frac{1}{2}(1 \pm \beta)
$$


and $s_{23}=\left(p_{2}+p_{3}\right)^{2} \equiv 2 k_{2} \cdot k_{3}$. The decomposition of eq. (2.5) has the advantage that momentum conservation is preserved, $p_{2}+p_{3}=k_{2}+k_{3}$. The inverse transformation is given by,

$$
\begin{aligned}
& k_{2}^{\mu}=\frac{1+\beta}{2 \beta} p_{2}^{\mu}-\frac{1-\beta}{2 \beta} p_{3}^{\mu}, \\
& k_{3}^{\mu}=\frac{1+\beta}{2 \beta} p_{3}^{\mu}-\frac{1-\beta}{2 \beta} p_{2}^{\mu} .
\end{aligned}
$$

In the rest of this paper we shall denote massive vectors by $p_{i},\left(p_{i}^{2} \neq 0\right)$ and massless vectors by $k_{i},\left(k_{i}^{2}=0\right)$. For the massless vectors $k_{i}$ we shall further define massless spinors,

$$
\begin{array}{ll}
\left.u_{-}\left(k_{i}\right)=|i-\rangle=\mid i\right], & u_{+}\left(k_{i}\right)=|i+\rangle=|i\rangle, \\
\bar{u}_{-}\left(k_{i}\right)=\langle i-|=\langle i|, & \bar{u}_{+}\left(k_{i}\right)=[i+\mid=[i \mid .
\end{array}
$$

In terms of the two massless momenta in eq. (2.7) the explicit results for the massive solutions of the Dirac equation are,

$$
\bar{u}_{ \pm}\left(p_{3}, m\right)=\frac{\beta_{+}^{-1 / 2}}{\left\langle 2^{\mp} \mid 3^{ \pm}\right\rangle}\left\langle 2^{\mp}\left|\left(\not \not_{3}+m\right), \quad v_{ \pm}\left(p_{2}, m\right)=\frac{\beta_{+}^{-1 / 2}}{\left\langle 2^{\mp} \mid 3^{ \pm}\right\rangle}\left(\not p_{2}-m\right)\right| 3^{ \pm}\right\rangle .
$$

This corresponds to choosing,

$$
\begin{aligned}
& \eta_{2}=k_{3}, \quad p_{2}^{b}=\beta_{+} k_{2}, \\
& \eta_{3}=k_{2}, \quad p_{3}^{b}=\beta_{+} k_{3},
\end{aligned}
$$

in eqs. $(2.2),(2.3)$.

With this choice, the results for the massive quark current with spin labels $\{+,-\}$ and $\{-,+\}$ have the same form as they would have in the massless limit (i.e. in the limit in which $p_{i} \rightarrow k_{i}$ for $\left.i=2,3\right)$,

$$
S^{\mu}\left(3_{Q}^{ \pm}, 2_{\bar{Q}}^{\mp}\right)=\bar{u}_{ \pm}\left(p_{3}, m\right) \gamma^{\mu} v_{\mp}\left(p_{2}, m\right)=\bar{u}_{ \pm}\left(k_{3}\right) \gamma^{\mu} v_{\mp}\left(k_{2}\right) \equiv\left\langle 3^{ \pm}\left|\gamma^{\mu}\right| 2^{ \pm}\right\rangle .
$$

The results for spin labels $\{-,-\}$ and $\{+,+\}$ on the heavy quark current are,

$$
\begin{aligned}
& S^{\mu}\left(3_{Q}^{-}, 2_{\bar{Q}}^{-}\right)=\bar{u}_{-}\left(p_{3}, m\right) \gamma^{\mu} v_{-}\left(p_{2}, m\right)=2 \mathcal{N}_{--}\left(k_{2}-k_{3}\right)^{\mu}, \\
& S^{\mu}\left(3_{Q}^{+}, 2_{\bar{Q}}^{+}\right)=\bar{u}_{+}\left(p_{3}, m\right) \gamma^{\mu} v_{+}\left(p_{2}, m\right)=2 \mathcal{N}_{++}\left(k_{2}-k_{3}\right)^{\mu},
\end{aligned}
$$

where the overall normalization is given by,

$$
\mathcal{N}_{--}=\frac{m}{[23]}, \quad \mathcal{N}_{++}=\frac{m}{\langle 23\rangle} .
$$

Contracting these equations with a Dirac matrix we obtain,

$$
\begin{aligned}
& S^{\mu}\left(3_{Q}^{+}, 2_{\bar{Q}}^{-}\right) \otimes\left\{\gamma_{\mu}\right\}=2\{|2\rangle[3|+| 3]\langle 2|\}, \\
& S^{\mu}\left(3_{Q}^{-}, 2_{\bar{Q}}^{+}\right) \otimes\left\{\gamma_{\mu}\right\}=2\{|3\rangle[2|+| 2]\langle 3|\}, \\
& S^{\mu}\left(3_{Q}^{-}, 2_{\bar{Q}}^{-}\right) \otimes\left\{\gamma_{\mu}\right\}=2 \mathcal{N}_{--}\{|2\rangle[2|-| 3\rangle[3|+| 2]\langle 2|-| 3]\langle 3|\}, \\
& S^{\mu}\left(3_{Q}^{+}, 2_{\bar{Q}}^{+}\right) \otimes\left\{\gamma_{\mu}\right\}=2 \mathcal{N}_{++}\{|2\rangle[2|-| 3\rangle[3|+| 2]\langle 2|-| 3]\langle 3|\} .
\end{aligned}
$$

As an example of the use of these spinors, in appendix B.1 we outline the calculation of the tree-level amplitudes for $g \bar{Q} Q g$ scattering, which will appear later as an ingredient in the calculation of our one-loop amplitudes. 

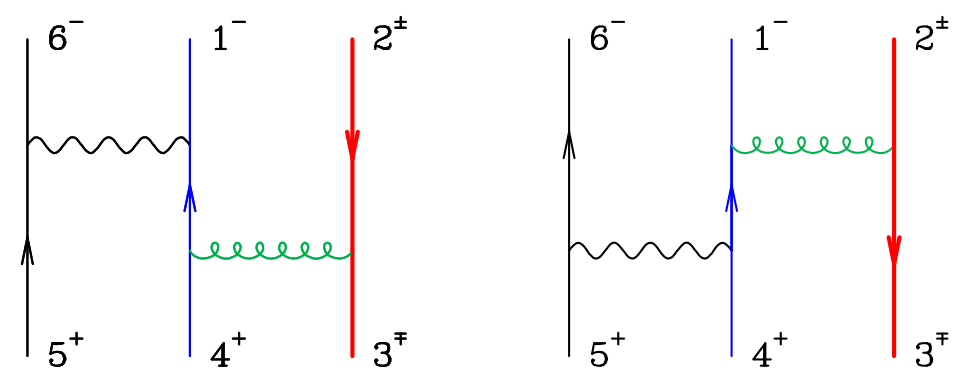

Figure 2. Feynman graphs that enter the calculation of the lowest order amplitude. The massive quarks are represented by the heavy (red) line, the wavy line denotes a $W$ boson and as usual the helical line denotes a gluon.

\section{Setup}

We shall consider the process,

$$
0 \rightarrow q\left(k_{1}\right)+\bar{Q}\left(p_{2}\right)+Q\left(p_{3}\right)+\bar{q}\left(k_{4}\right)+\bar{\ell}\left(k_{5}\right)+\ell\left(k_{6}\right)
$$

both at tree level and including the one-loop QCD corrections. The process is mediated by the exchange of a $W$-boson which decays into an antilepton-lepton pair with momenta labelled by $k_{5}$ and $k_{6}$, as shown in figure 2 . The V-A structure of the charged weak interaction ensures that the lepton and massless-quark lines will have fixed helicity. Thus the outgoing lepton (6) will be always left-handed and the outgoing antilepton (5) always right-handed. With this understanding we can often drop the lepton labels from the specification of the amplitude.

\subsection{Colour decomposition}

As noted above we shall suppress the labels for the outgoing leptons 5 and 6 which play no role in the colour decomposition. The colour decomposition for the tree graphs is,

$$
\mathcal{A}_{6}^{\text {tree }}\left(1_{q}, 2_{\bar{Q}}, 3_{Q}, 4_{\bar{q}}\right)=g_{W}^{2} g^{2} \mathcal{P}_{W}\left(s_{56}\right) A_{6}^{\text {tree }}\left(1_{q}, 2_{\bar{Q}}, 3_{Q}, 4_{\bar{q}}\right)\left(\delta_{j_{1}}^{\bar{j}_{2}} \delta_{j_{3}}^{\bar{j}_{4}}-\frac{1}{N_{c}} \delta_{j_{1}}^{\bar{j}_{4}} \delta_{j_{3}}^{\bar{j}_{2}}\right)
$$

In eq. (3.2) we have introduced the strong coupling constant, $g$, and the weak coupling, $g_{W}$, defined through,

$$
\alpha_{S}=\frac{g^{2}}{4 \pi}, \quad \frac{G_{F}}{\sqrt{2}}=\frac{g_{W}^{2}}{8 M_{W}^{2}} .
$$

The Breit-Wigner factor $\mathcal{P}_{W}(s)$ is given by,

$$
\mathcal{P}_{W}(s)=\frac{s}{s-M_{W}^{2}+i \Gamma_{W} M_{W}} .
$$

The indices $j_{1}, j_{3},\left(\bar{j}_{2}, \bar{j}_{4}\right)$ denote the colour labels of the corresponding quark (antiquark) lines. 
At one loop the colour decomposition is given by,

$$
\begin{aligned}
\mathcal{A}_{6}^{1-\text { loop }} & \left(1_{q}, 2_{\bar{Q}}, 3_{Q}, 4_{\bar{q}}\right)=g_{W}^{2} g^{4} c_{\Gamma} \mathcal{P}_{W}\left(s_{56}\right) \\
& \times\left[N_{c} \delta_{j_{1}}^{\bar{j}_{2}} \delta_{j_{3}}^{\bar{j}_{4}} A_{6 ; 1}\left(1_{q}, 2_{\bar{Q}}, 3_{Q}, 4_{\bar{q}}\right)+\delta_{j_{1}}^{\bar{j}_{4}} \delta_{j_{3}}^{\bar{j}_{2}} A_{6 ; 2}\left(1_{q}, 2_{\bar{Q}}, 3_{Q}, 4_{\bar{q}}\right)\right],
\end{aligned}
$$

where the overall factor $c_{\Gamma}$ is,

$$
c_{\Gamma}=\frac{1}{(4 \pi)^{2-\epsilon}} \frac{\Gamma(1+\epsilon) \Gamma^{2}(1-\epsilon)}{\Gamma(1-2 \epsilon)} .
$$

The interference of the one-loop amplitude with the lowest order, summed over initial and final colours, is given by,

$$
\begin{aligned}
\sum_{\text {colours }}\left[\mathcal{A}_{6}^{*} \mathcal{A}_{6}\right]_{\mathrm{NLO}}=2 g_{W}^{4} g^{6} c_{\Gamma}\left(N_{c}^{2}-1\right) N_{c}\left|\mathcal{P}_{W}\left(s_{56}\right)\right|^{2} \\
\times \operatorname{Re}\left\{\left[A_{6}^{\text {tree }}\left(1_{q}, 2,3,4_{\bar{q}}\right)\right]^{*} A_{6 ; 1}\left(1_{q}, 2,3,4_{\bar{q}}\right)\right\} .
\end{aligned}
$$

Therefore $A_{6 ; 2}$ plays no role in the calculation of NLO corrections.

\subsection{Tree level amplitudes}

The tree level amplitudes can be calculated using the diagrams of figure 2 . The $\{-+\}$ amplitude, expressed in terms of the momenta $k_{i}$, is identical to the massless result as a consequence of eq. (2.11),

$$
\begin{aligned}
-i A_{6}^{\text {tree }}\left(1_{q}^{-}, 2_{\bar{Q}}^{+}, 3_{Q}^{-}, 4_{\bar{q}}^{+}, 5_{\bar{\ell}}^{+}, 6_{\ell}^{-}\right) & =\left[\frac{\langle 13\rangle[45]\langle 6|(1+3)| 2]}{s_{23} s_{56} s_{123}}-\frac{[42]\langle 16\rangle[5|(2+4)| 3\rangle}{s_{23} s_{56} s_{234}}\right] \\
& \equiv\left[\left\{\frac{\langle 13\rangle[45]\langle 6|(1+3)| 2]}{s_{23} s_{56} s_{123}}\right\}-\{\text { flip }\}\right]
\end{aligned}
$$

where we have introduced the symmetry,

$$
\text { flip : }(1 \leftrightarrow 4),(2 \leftrightarrow 3),(5 \leftrightarrow 6),[] \leftrightarrow\langle\rangle \text {. }
$$

The $\{--\}$ amplitude is given by,

$$
\begin{aligned}
-i A_{6}^{\text {tree }}\left(1_{q}^{-}\right. & \left., 2_{\bar{Q}}^{-}, 3_{Q}^{-}, 4_{\bar{q}}^{+}, 5_{\bar{\ell}}^{+}, 6_{\ell}^{-}\right)=\mathcal{N}_{--} \\
\times & {\left[\left\{[45]\left(\frac{\langle 6|(1+2)| 3]\langle 31\rangle-\langle 6|(1+3)| 2]\langle 21\rangle}{s_{23} s_{56} s_{123}}\right)\right\}+\{\text { flip }\}\right], }
\end{aligned}
$$

with the mass-dependent normalization factor given in eq. (2.13). The amplitudes for $A_{6}^{\text {tree }}\left(1^{-}, 2^{-}, 3^{+}, 4^{+}, 6^{-}, 5^{+}\right)$and $A_{6}^{\text {tree }}\left(1^{-}, 2^{+}, 3^{+}, 4^{+}, 6^{-}, 5^{+}\right)$can be easily obtained by reference to eqs. (2.11), (2.12),

$$
\begin{aligned}
& A_{6}^{\text {tree }}\left(1_{q}^{-}, 2_{\bar{Q}}^{-}, 3_{Q}^{+}, 4_{\bar{q}}^{+}, 5_{\bar{\ell}}^{+}, 6_{\ell}^{-}\right)=A_{6}^{\text {tree }}\left(1_{q}^{-}, 3_{\bar{Q}}^{+}, 2_{Q}^{-}, 4_{\bar{q}}^{+}, 5_{\bar{\ell}}^{+}, 6_{\ell}^{-}\right), \\
& A_{6}^{\text {tree }}\left(1_{q}^{-}, 2_{\bar{Q}}^{+}, 3_{Q}^{+}, 4_{\bar{q}}^{+}, 5_{\bar{\ell}}^{+}, 6_{\ell}^{-}\right)=- \text {flip }\left[A_{6}^{\text {tree }}\left(1_{q}^{-}, 2_{\bar{Q}}^{-}, 3_{Q}^{-}, 4_{\bar{q}}^{+}, 5_{\bar{\ell}}^{+}, 6_{\ell}^{-}\right)\right] .
\end{aligned}
$$



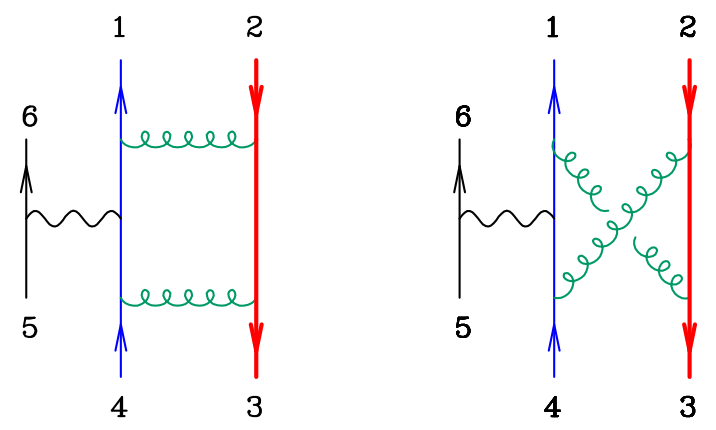

Figure 3. Parent diagrams for the leading colour primitive $A_{6}^{\mathrm{lc}}(1,2,3,4)$ and crossed box primitive $A_{6}^{\mathrm{cb}}(1,2,3,4)$.

\subsection{Decomposition of one-loop amplitudes into primitive amplitudes}

Following ref. [18] we can decompose the full one-loop amplitude into gauge invariant primitive amplitudes,

$$
\begin{aligned}
A_{6 ; 1}\left(1_{q}, 2_{\bar{Q}}, 3_{Q}, 4_{\bar{q}}\right)= & A_{6}^{\mathrm{lc}}(1,2,3,4)-\frac{2}{N^{2}}\left(A_{6}^{\mathrm{cb}}(1,2,3,4)+A_{6}^{\mathrm{lc}}(1,2,3,4)\right) \\
& -\frac{1}{N^{2}} A_{6}^{\mathrm{sl}}(1,2,3,4)-\frac{n_{\mathrm{lf}}}{N} A_{6}^{\mathrm{lf}}(1,2,3,4)-\frac{n_{\mathrm{hf}}}{N} A_{6}^{\mathrm{hf}}(1,2,3,4), \\
A_{6 ; 2}\left(1_{q}, 2_{\bar{Q}}, 3_{Q}, 4_{\bar{q}}\right)= & A_{6}^{\mathrm{cb}}(1,2,3,4)+\frac{1}{N^{2}}\left(A_{6}^{\mathrm{lc}}(1,2,3,4)+A_{6}^{\mathrm{cb}}(1,2,3,4)\right) \\
& +\frac{1}{N^{2}} A_{6}^{\mathrm{sl}}(1,2,3,4)+\frac{n_{\mathrm{lf}}}{N} A_{6}^{\mathrm{lf}}(1,2,3,4)+\frac{n_{\mathrm{hf}}}{N} A_{6}^{\mathrm{hf}}(1,2,3,4) .
\end{aligned}
$$

$A_{6}^{\text {lc }}$ is the primitive amplitude containing the leading colour box and $A_{6}^{\mathrm{cb}}$ is the primitive amplitude containing the crossed box. The parent diagrams are shown in figure 3 . These two primitive amplitudes are related by,

$$
A_{6}^{\mathrm{cb}}\left(1_{q}^{h_{1}}, 2_{\bar{Q}}^{h_{2}}, 3_{Q}^{h_{3}}, 4_{\bar{q}}^{h_{4}}\right)=-A_{6}^{\mathrm{lc}}\left(1_{q}^{h_{1}}, 3_{\bar{Q}}^{h_{3}}, 2_{Q}^{h_{2}}, 4_{\bar{q}}^{h_{4}}\right) .
$$

$A_{6}^{\text {sl }}$ is the primitive amplitude containing the subleading colour boxes, see figure 4 , and the primitive amplitude for the fermion-loop diagrams (light and heavy), $A_{6}^{\mathrm{lf}}, A_{6}^{\mathrm{hf}}$ is shown in figure 5. We note that, just as at tree level, parity ensures that we need only compute a reduced set of amplitudes. For $A_{6}^{\text {lc }}$ at one loop it is sufficient to calculate three of the four possible spin label combinations since (c.f. eq. 3.12),

$$
A_{6}^{\mathrm{lc}}\left(1_{q}^{-}, 2_{\bar{Q}}^{+}, 3_{Q}^{+}, 4_{\bar{q}}^{+}, 5_{\bar{\ell}}^{+}, 6_{\ell}^{-}\right)=- \text {flip }\left[A_{6}^{\mathrm{lc}}\left(1_{q}^{-}, 2_{\bar{Q}}^{-}, 3_{Q}^{-}, 4_{\bar{q}}^{+}, 5_{\bar{\ell}}^{+}, 6_{\ell}^{-}\right)\right] .
$$

\subsection{Structure of the calculation}

In the presence of propagators with vanishing masses on internal lines the one-loop amplitude contains infrared and collinear divergences. In addition, the amplitude contains ultraviolet divergences. We regulate all of these divergences using dimensional regularization, continuing the loop integration to $D=4-2 \epsilon$ dimensions. The divergences then 

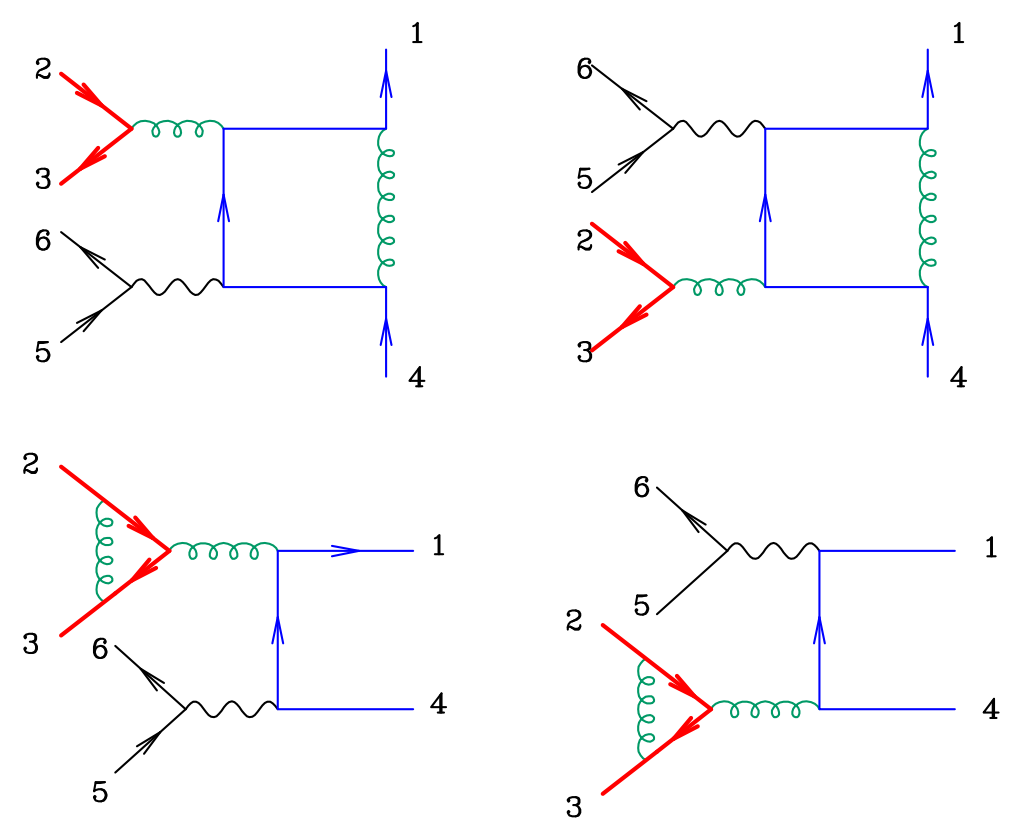

Figure 4. Parent diagrams for the subleading colour primitive amplitude, $A_{6}^{\mathrm{sl}}(1,2,3,4)$.
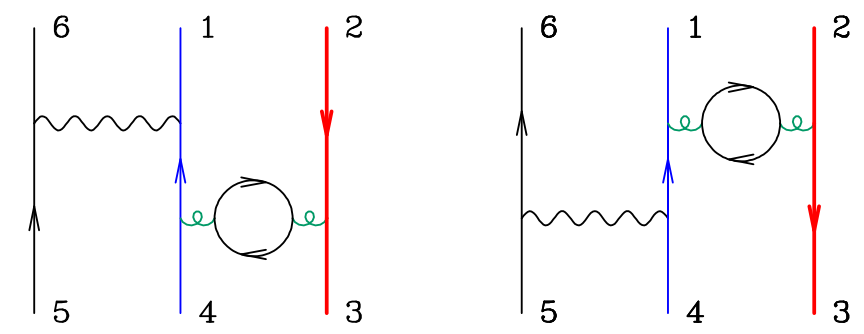

Figure 5. Diagrams for the fermion loop primitives $A_{6}^{\mathrm{lf}}(1,2,3,4)$ and $A_{6}^{\mathrm{hf}}(1,2,3,4)$.

appear as poles in $\epsilon$. For the primitive amplitudes the divergence structure is quite simple and we can separate the amplitude as follows,

$$
A_{6}^{j}=\left[A_{6}^{\mathrm{tree}} V^{j}+i F^{j}\right]
$$

where $V^{j}$ contains all the divergent pieces. In this equation $A_{6}^{j}$ is any of the primitive amplitudes and the index $j$ runs over $\{\mathrm{lc}, \mathrm{cb}, \mathrm{sl}, \mathrm{lf}, \mathrm{hf}\}$. As we shall see later the simplicity of the pole structure gives useful constraints on the different terms contributing to the amplitude.

The calculation proceeds by noting that the primitive amplitudes can be expressed as a sum of scalar box, triangle, bubble and tadpole integrals and a rational part,

$$
\begin{aligned}
& -i A_{6}^{j}\left(1^{-}, 2^{h_{2}}, 3^{h_{3}}, 4^{+}, 5^{+}, 6^{-}\right)= \\
& \sum_{i_{1} i_{2} i_{3}} d_{i_{1}\left|i_{2}\right| i_{3}}\left(h_{3}, h_{2}\right) D_{0}\left(p_{i_{1}}, p_{i_{2}}, p_{i_{3}}\right)+\sum_{i_{1} i_{2}} c_{i_{1} \mid i_{2}}\left(h_{3}, h_{2}\right) C_{0}\left(p_{i_{1}}, p_{i_{2}}\right) \\
& \quad+\sum_{i_{1}} b_{i_{1}}\left(h_{3}, h_{2}\right) B_{0}\left(p_{i_{1}}\right)+a\left(h_{3}, h_{2}\right) A_{0}+R^{j}\left(1^{-}, 2^{h_{2}}, 3^{h_{3}}, 4^{+}, 5^{+}, 6^{-}\right),
\end{aligned}
$$




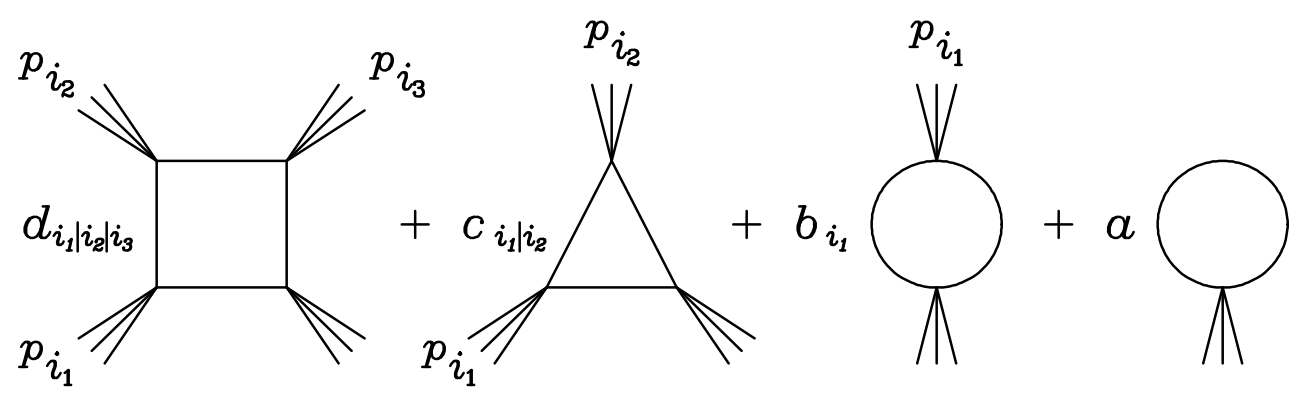

Figure 6. Schematic diagram illustrating the decomposition in scalar integrals.

where the label $j$ runs over the values $\{\mathrm{lc}, \mathrm{cb}, \mathrm{sl}, \mathrm{lf}, \mathrm{hf}\}$. In this equation, illustrated in figure 6 , the indices $i_{1}, i_{2}, i_{3}$ run over all partitions of the cyclically ordered momenta. The coefficients $d, c, b, a$ of the scalar integrals in eq. (3.17) also depend on the primitive amplitude index $j$. In the following we shall suppress this dependence, but the primitive amplitude to which we are referring will be clear from the context. The functions $A_{0}, B_{0}, C_{0}, D_{0}$ are the scalar integrals defined in appendix C. The mass labels have been suppressed in the scalar integrals in eq. (3.17) because they are determined automatically by the propagators connecting the two external massive quarks. Values for these scalar integrals are obtained using the QCDLoop program [19]. By using four-dimensional unitarity methods $[10-13,20]$, we obtain the coefficients, $a, b, c, d$ in the four-dimensional helicity (FDH) scheme [21]. The rational terms require information from beyond four dimensions. Both of these calculations are performed making extensive use of the algebraic manipulation program Form [22].

\section{The results for primitive amplitude $A_{6}^{\text {lc }}$}

\subsection{Divergent parts}

For the leading colour primitive amplitude the divergent term which enters in eq. (3.16) is given by [23],

$$
V^{l c}=-\frac{1}{\epsilon^{2}}\left[\left(\frac{\mu^{2}}{-2 k_{4} \cdot p_{3}}\right)^{\epsilon}+\left(\frac{\mu^{2}}{-2 k_{1} \cdot p_{2}}\right)^{\epsilon}-\left(\frac{\mu^{2}}{m^{2}}\right)^{\epsilon}\right]+\frac{8}{3} \frac{1}{\epsilon} .
$$

\subsection{Calculation of the box coefficients}

The five scalar box integrals that enter the leading colour amplitude are enumerated in table 1 . Of these, we directly calculate the coefficients of boxes 1-3 and obtain the remaining two by symmetry:

$$
\begin{aligned}
d_{2|3| 4}\left(h_{3}, h_{2}\right) & =- \text { flip }\left[d_{1|2| 3}\left(-h_{2},-h_{3}\right)\right], \\
d_{12|3| 4}\left(h_{3}, h_{2}\right) & =- \text { flip }\left[d_{1|2| 34}\left(-h_{2},-h_{3}\right)\right],
\end{aligned}
$$

where $h_{3}$ and $h_{2}$ represent the spin labels of the quark and antiquark respectively and the operation flip is defined in eq. (3.9). When using the Rodrigo choice to decompose $p_{2}$ and $p_{3}$, box 3 only gives a non-zero contribution for the spin-labels $\{-,-\}$ and $\{+,+\}$. 


\begin{tabular}{|l|c|c|c|}
\hline Scalar integral & Coefficient & Scalar integral & Coefficient \\
\hline 1. $D_{0}\left(k_{1}, p_{2}, p_{3} ; 0,0, m, 0\right)$ & $d_{1|2| 3}$ & 4. $D_{0}\left(p_{2}, p_{3}, k_{4} ; 0, m, 0,0\right)$ & $d_{2|3| 4}$ \\
2. $D_{0}\left(k_{1}, p_{2}, p_{34} ; 0,0, m, 0\right)$ & $d_{1|2| 34}$ & 5. $D_{0}\left(p_{12}, p_{3}, k_{4} ; 0, m, 0,0\right)$ & $d_{12|3| 4}$ \\
3. $D_{0}\left(k_{1}, p_{23}, k_{4} ; 0,0,0,0\right)$ & $d_{1|23| 4}$ & & \\
\hline
\end{tabular}

Table 1. Scalar box integrals appearing in the leading colour amplitude $A_{6}^{l c}(1,2,3,4)$ and the notation used in the text to denote their coefficients.

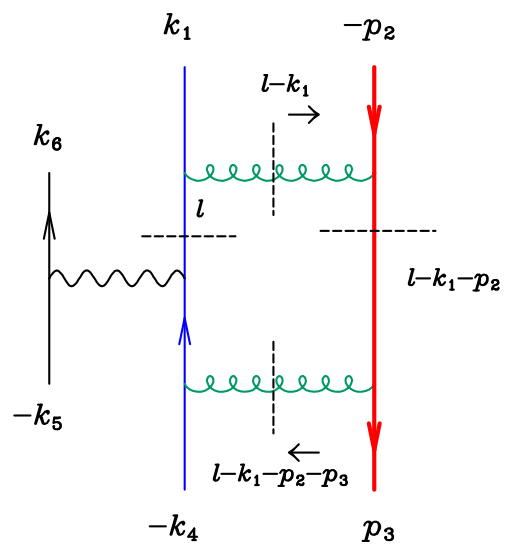

Figure 7. Diagram for the calculation of the coefficient of the scalar integral $D_{0}\left(k_{1}, p_{2}, p_{3} ; 0,0, m, 0\right)$. The massive quark is represented by the heavy (red) line and unitarity cuts are represented by dashed lines.

The coefficients are computed using quadruple cuts with complex momenta [10]. For boxes 1 and 2 the presence of a massive propagator leads to a more complicated parametrization of the loop momentum than in the purely massless case. The particular parameterizations that we have used are spelled out below.

\subsubsection{Calculation of $d_{1|2| 3}$}

The cuts used to isolate this coefficient are depicted in figure 7 , where we remind the reader that we work in the case that $k_{1}^{2}=k_{4}^{2}=0$ and $p_{2}^{2}=p_{3}^{3}=m^{2}$. The loop momentum is subject to the following constraints,

$$
l^{2}=0, \quad\left(l-k_{1}\right)^{2}=0, \quad\left(l-k_{1}-p_{2}\right)^{2}=m^{2}, \quad\left(l-k_{1}-p_{2}-p_{3}\right)^{2}=0,
$$

Denoting $l_{1}=l-k_{1}$ we have $l_{1} \cdot k_{1}=0$ so we must have either $\left|l_{1}\right\rangle \sim|1\rangle$ or $\left.\left.\mid l_{1}\right] \sim \mid 1\right]$, depending on the helicity of the internal gluons, in such a way that the 3-point $q \bar{q} g$ vertex is non-vanishing.

In the case $\left[l_{1} \mid \sim[1 \mid\right.$ we can write

$$
\begin{aligned}
& l_{1}^{\mu}=l^{\mu}-k_{1}^{\mu}=\frac{s_{23}}{2} \frac{\left[1\left|\gamma^{\mu} \mathbf{2}\right| 1\right]}{[1|(\mathfrak{Z}+\boldsymbol{3}) \mathfrak{Z}| 1]} \equiv \frac{s_{23}}{2} \frac{\left[1\left|\gamma^{\mu} \mathbf{2}\right| 1\right]}{[1|\mathfrak{3} \mathbf{Z}| 1]}, \\
& l_{123}^{\mu}=l_{1}^{\mu}-p_{2}^{\mu}-p_{3}^{\mu}=-\frac{1}{2} \frac{\left[1\left|(\mathbf{2}+\boldsymbol{3}) \gamma^{\mu}(\mathbf{2}+\mathbf{3}) \mathbf{2}\right| 1\right]}{[1|\mathbf{3 2}| 1]},
\end{aligned}
$$


where the boldface momenta denote massive vectors. By inspection, it is clear that this parametrization of the loop momentum $l^{\mu}$ satisfies the constraints in eq. (4.3). Correspondingly in the case $\left\langle l_{1}\right| \sim\langle 1|$ we can write

$$
\begin{aligned}
& l_{1}^{\mu}=l^{\mu}-k_{1}^{\mu}=\frac{s_{23}}{2} \frac{\left\langle 1\left|\mathfrak{2} \gamma^{\mu}\right| 1\right\rangle}{\langle 1|\mathfrak{2}(\mathfrak{2}+\boldsymbol{Z})| 1\rangle} \equiv \frac{s_{23}}{2} \frac{\left\langle 1\left|\mathfrak{2} \gamma^{\mu}\right| 1\right\rangle}{\langle 1|\mathfrak{2} \boldsymbol{\not}| 1\rangle}, \\
& l_{123}^{\mu}=l_{1}^{\mu}-p_{2}^{\mu}-p_{3}^{\mu}=-\frac{1}{2} \frac{\left\langle 1\left|\boldsymbol{2}(\mathfrak{2}+\boldsymbol{Z}) \gamma^{\mu}(\mathfrak{2}+\boldsymbol{Z})\right| 1\right\rangle}{\langle 1|\mathfrak{2} \boldsymbol{\not}| 1\rangle} .
\end{aligned}
$$

Note that the denominator in eq. (4.5) simplifies when expressed in terms of massless momenta,

$$
\langle 1|\mathbf{2} \boldsymbol{3}| 1\rangle=\beta\langle 12\rangle[23]\langle 31\rangle,
$$

As an example, we will calculate the coefficient of this box integral for the spin label choice $\{-,+\}$. The box coefficient is given by the formula,

$$
\begin{aligned}
d_{1|2| 3}(-,+) \equiv & \frac{1}{2} \sum_{h_{a}, h_{b}} d_{1|2| 3}^{\left[h_{a}, h_{b}\right]}(-,+) \\
= & \frac{1}{2} \sum_{h_{a}, h_{b}} A_{3}\left(1_{q}^{-},-l_{\bar{q}}^{+}, l_{1}^{h_{a}}\right) \times A_{5}\left(l_{q}^{-}, 4_{\bar{q}}^{+},-l_{123}^{h_{b}}, 5_{\bar{l}}^{+}, 6_{l}^{-}\right) \\
& \quad \times A_{4}\left(-l_{1}^{-h_{a}}, 2_{\bar{Q}}^{+}, 3_{Q}^{-}, l_{123}^{-h_{b}}\right) \times(-i)\left[\left(l-k_{1}-p_{2}\right)^{2}-m^{2}\right],
\end{aligned}
$$

with the loop momenta fixed according to the constraints above, i.e. $l^{\mu}$ is given by either eq. (4.4) or (4.5) above. In this formula we have for brevity suppressed the gluon particle labels. Note that we have not explicitly cut the heavy quark propagator but, equivalently, have simply written the full $g \bar{Q} Q g$ amplitude and multiplied by the cut propagator. Since the $g \bar{Q} Q g$ amplitudes with the same helicity gluons vanish (c.f. eq. (B.13)), the coefficient receives only two contributions.

Let us first inspect the assignment $h_{a}=-h_{b}=+1$. We note that it is simplest to manipulate the $g \bar{Q} Q g$ amplitude, (eq. (B.13) first line), using the Schouten identity, eq. (A.6), into an alternative form in order to take full advantage of the vanishing of the cut propagator,

$$
\begin{aligned}
-i A_{4}\left(-l_{1}^{-}, 2_{\bar{Q}}^{+}, 3_{Q}^{-}, l_{123}^{+}\right)\left[\left(l_{1}-p_{2}\right)^{2}-m^{2}\right] & =\frac{\left[2 l_{123}\right]^{2}\left\langle l_{1}|\mathbf{2}| l_{123}\right]}{\left[l_{1} l_{123}\right][23]} \\
& =-\frac{\left[2 l_{123}\right]^{2}\left(\left[l_{1} l_{123}\right]\left\langle l_{1}|\boldsymbol{2}| 3\right]-\left\langle l_{1}|\boldsymbol{2}| l_{1}\right]\left[3 l_{123}\right]\right)}{\left[l_{1} 3\right]\left[l_{1} l_{123}\right][23]} \\
& =-\frac{\left[2 l_{123}\right]^{2}}{\left[l_{1} 3\right][23]}\left(\left\langle l_{1}|\boldsymbol{2}| 3\right]-\frac{\left[3 l_{123}\right]\left\langle l_{1}|\boldsymbol{2}| l_{1}\right]}{\left[l_{1} l_{123}\right]}\right) .
\end{aligned}
$$

On shell, $\left(l_{1}-p_{2}\right)^{2}-m^{2} \equiv\left\langle l_{1}|\boldsymbol{2}| l_{1}\right]=0$ and we can simply discard the second term in this equation. Using results for the other amplitudes presented in appendix B we find that,

$$
d_{1|2| 3}^{[+,-]}(-,+)=\frac{\left[l l_{1}\right]^{2}}{[l 1]} \times \frac{[45]^{2}}{\left[4 l_{123}\right]\left[l l_{123}\right][65]} \times \frac{\beta_{+}\left[2 l_{123}\right]^{2}\left\langle 2 l_{1}\right\rangle}{\left[l_{1} 3\right]}
$$


where we have simplified the $g \bar{Q} Q g$ amplitude further using the decomposition of $p_{2}$ in terms of $k_{2}$ and $k_{3}$. Since the propagator $l$ does not have a simple decomposition in terms of external momenta, it is simplest to eliminate it by multiplying this expression in numerator and denominator by a factor $\langle 4 l\rangle\left\langle l_{123} l\right\rangle$. After some simplification this yields,

$$
d_{1|2| 3}^{[+,-]}(-,+)=\frac{\beta_{+}[45]^{2}\langle 41\rangle}{s_{123}[65]} \frac{\left\langle l_{123} 1\right\rangle\left[1 l_{1}\right]\left[2 l_{123}\right]^{2}\left\langle 2 l_{1}\right\rangle}{\left\langle 4 l_{1}\right\rangle\left[4 l_{123}\right]\left[l_{1} 3\right]},
$$

This is now in a form where we can use the loop momenta definitions given in eq. (4.5) with spinors identified by, for example, using the identity, $l^{\mu}=\frac{1}{2}\left\langle l\left|\gamma^{\mu}\right| l\right]$. We can thus make the replacements:

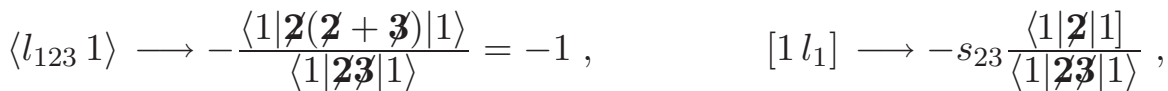

$$
\begin{aligned}
& {\left[4 l_{123}\right] \longrightarrow[4|(\boldsymbol{2}+\boldsymbol{3})| 1\rangle=\langle 1|2+3| 4], \quad\left[2 l_{123}\right] \longrightarrow[2|(\boldsymbol{2}+\boldsymbol{Z})| 1\rangle=[23]\langle 31\rangle,} \\
& \left\langle 2 l_{1}\right\rangle \longrightarrow\langle 21\rangle, \quad\left\langle 4 l_{1}\right\rangle \longrightarrow\langle 41\rangle, \\
& {\left[l_{1} 3\right] \longrightarrow s_{23} \frac{\langle 1|\mathfrak{2}| 3]}{\langle 1|\mathfrak{2} \mathbf{3}| 1\rangle}=s_{23} \frac{\beta_{+}\langle 12\rangle[23]}{\langle 1|\mathbf{2 3}| 1\rangle} .}
\end{aligned}
$$

This results in the final expression,

$$
d_{1|2| 3}^{[+,-]}(-,+)=\frac{\langle 13\rangle^{2}[23][45]^{2}\langle 1|2| 1]}{s_{123}\langle 1|2+3| 4][56]} .
$$

Adding in the contribution from the other helicity configuration $\left(h_{a}=-h_{b}=-1\right)$, computed in a similar fashion, yields the full result for this coefficient,

$$
d_{1|2| 3}(-,+)=\frac{s_{23}\langle 1|\boldsymbol{2}| 1]}{2 s_{123}}\left[\frac{[23]}{\langle 56\rangle\langle 4|2+3| 1]}\left(\frac{\langle 6|\mathfrak{z}| 1]}{\beta[13]}+\frac{\langle 16\rangle[12]}{[23]}\right)^{2}-\frac{\langle 13\rangle^{2}[45]^{2}}{\langle 23\rangle[56]\langle 1|2+3| 4]}\right] .
$$

This is in agreement with the coefficient presented in ref. [18] in the limit that the heavy quark mass is taken to zero.

\subsubsection{Calculation of $d_{1|2| 34}$}

This coefficient is obtained from the cuts shown in figure 8 , where the loop momentum is subject to the conditions,

$$
l^{2}=0, \quad\left(l-k_{1}\right)^{2}=0, \quad\left(l-k_{1}-p_{2}\right)^{2}=m^{2}, \quad l_{1234}^{2}=\left(l-k_{1}-p_{2}-p_{3}-k_{4}\right)^{2}=0 .
$$

An explicit solution for $l_{1}=l-k_{1}$ is given by,

$$
l_{1}^{\mu}=\frac{s_{234}}{2} \frac{\left\langle 1\left|\mathfrak{2} \gamma^{\mu}\right| 1\right\rangle}{\langle 1|\mathfrak{2}(\not{3}+\not{\not})| 1\rangle},
$$

which clearly satisfies the constraints in eq. (4.14). From this we can also derive a useful form for the momentum $l_{1234}$,

$$
l_{1234}^{\mu}=l_{1}-p_{2}-p_{3}-k_{4}=-\frac{1}{2} \frac{\left\langle 1\left|\mathfrak{Z}(\not{2}+\not{3}+\not{4}) \gamma^{\mu}(\mathfrak{2}+\not{3}+\not{\not})\right| 1\right\rangle}{\langle 1|\boldsymbol{2}(\not{3}+\not{\not})| 1\rangle} .
$$




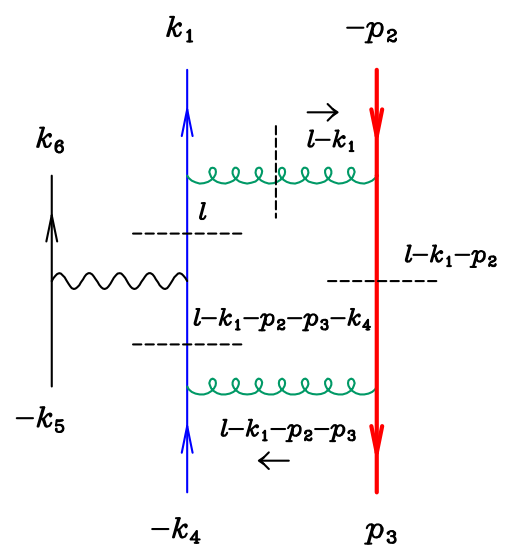

Figure 8. Diagram for the calculation of the coefficient of the scalar integral $D_{0}\left(k_{1}, p_{2}, p_{34} ; 0,0, m, 0\right)$. The massive quark is represented by the heavy (red) line and unitarity cuts are represented by dashed lines.

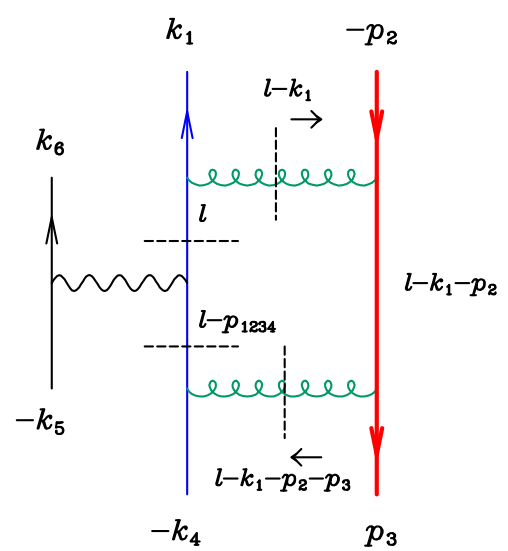

Figure 9. Diagram for the calculation of the coefficient of the scalar integral $D_{0}\left(k_{1}, p_{23}, k_{4} ; 0,0,0,0\right)$. The massive quark is represented by the heavy (red) line and unitarity cuts are represented by dashed lines.

The other solution, corresponding to $\left[l_{1} \mid \sim[1 \mid\right.$ can be obtained from eq. (4.15) by complex conjugation. We note that both of these parameterizations can be obtained from eqs. (4.4) and (4.5) by simply making the replacements, $\not{3} \rightarrow(\boldsymbol{3}+\not{4})$ and $s_{23} \rightarrow s_{234}$. From these forms it is straightforward to compute the coefficients of this box.

\subsubsection{Calculation of $d_{1|23| 4}$}

The cuts used to isolate this coefficient are depicted in figure 9. This box with massless internal lines and two opposite massive external lines is often referred to as the easy box [24]. The heavy quark does not participate in the loop, and the loop momentum is subject to the following constraints,

$$
l^{2}=0, \quad\left(l-k_{1}\right)^{2}=0, \quad\left(l-k_{1}-p_{2}-p_{3}\right)^{2}=0, \quad\left(l-k_{1}-p_{2}-p_{3}-k_{4}\right)^{2}=0,
$$


The explicit solutions for $l_{1}=l-k_{1}$ and $l_{123}=l-k_{1}-p_{2}-p_{3}$ are given either by,

$$
\begin{aligned}
l_{1}^{\mu} & =\frac{1}{2} \frac{\left\langle 4\left|(\boldsymbol{Z}+\boldsymbol{Z}) \gamma^{\mu}\right| 1\right\rangle}{\langle 41\rangle}, \\
l_{123}^{\mu} & =-\frac{1}{2} \frac{\left\langle 4\left|\gamma^{\mu}(\mathbf{2}+\boldsymbol{3})\right| 1\right\rangle}{\langle 41\rangle},
\end{aligned}
$$

or by,

$$
\begin{aligned}
l_{1}^{\mu} & =\frac{1}{2} \frac{\left[4\left|(\boldsymbol{2}+\mathbf{3}) \gamma^{\mu}\right| 1\right]}{[41]}, \\
l_{123}^{\mu} & =-\frac{1}{2} \frac{\left[4\left|\gamma^{\mu}(\mathbf{2}+\boldsymbol{3})\right| 1\right]}{[41]} .
\end{aligned}
$$

Both of these clearly satisfy the constraints in eq. (4.17).

As an example, we will calculate the coefficient of this box integral for the spin label choice $\{-,+\}$. For this spin label choice it turns out that the box coefficient vanishes. The argument is quite general and may be useful in explaining the absence of the easy box in other contexts, e.g. ref. [25]. The result for the box coefficient is calculated from,

$$
\begin{aligned}
d_{1|23| 4}(-,+)=\sum_{h_{a}, h_{b}} A_{3}\left(1_{q}^{-},-l^{+}{ }_{\bar{q}}, l_{1}^{h_{a}}\right) \times A_{4}\left(l^{-}{ }_{q},-l_{1234 \bar{q}}^{+}, 5_{\bar{l}}^{+}, 6_{l}^{-}\right) \\
\times A_{3}\left(l_{1234 q}^{-}, 4_{\bar{q}}^{+},-l_{123}^{h_{b}}\right) \times A_{4}\left(-l_{1}^{-h_{a}}, 2_{\bar{Q}}^{+}, 3_{Q}^{-}, l_{123}^{-h_{b}}\right),
\end{aligned}
$$

with the loop momenta fixed according to the constraints above, i.e. $l^{\mu}$ is given by either eq. (4.18) or (4.19) above. From eq. (B.13) we see that $A_{4}\left(-l_{1}^{-h_{a}}, 2_{\bar{Q}}^{+}, 3_{Q}^{-}, l_{123}^{-h_{b}}\right)$ vanishes for $h_{a}=h_{b}$. Therefore we need only consider the case $h_{a}=-h_{b}$. For definiteness let us consider the case $h_{a}=1, h_{b}=-1$. For this case we find that,

$$
\begin{aligned}
& A_{3}\left(1_{q}^{-},-l^{+}, l_{1}^{+}\right)=\frac{\left[l l_{1}\right]^{2}}{[1 l]\left[l_{1} 1\right]} \Longrightarrow\left|l_{1}\right\rangle \sim|1\rangle, \\
&\left.\left.A_{3}\left(l_{1234 q}^{-}, 4_{\bar{q}}^{+},-l_{123}^{-}\right)=\frac{\left\langle l_{1234} l_{123}\right\rangle^{2}}{\left\langle 4 l_{123}\right\rangle\left\langle 4 l_{1234}\right\rangle} \quad \Longrightarrow \mid l_{123}\right] \sim \mid 4\right] .
\end{aligned}
$$

However we see that the conditions $\left.\left.\left|l_{1}\right\rangle \sim|1\rangle, \mid l_{123}\right] \sim \mid 4\right]$ in eq. (4.21) are not compatible with the kinematic constraints, eqs. (4.18), (4.19) which require either,

$$
\begin{aligned}
\left|l_{1}\right\rangle & \sim|1\rangle \text { and }\left|l_{123}\right\rangle \sim|4\rangle, \\
\text { or } \left.\mid l_{1}\right] & \left.\left.\sim \mid 1] \text { and } \mid l_{123}\right] \sim \mid 4\right],
\end{aligned}
$$

respectively. The argument follows identically for the contribution $h_{a}=-1, h_{b}=+1$. Therefore for this external spin label choice $\{-,+\}$ the box coefficient is zero. For the spin label choice $\{-,-\}$ there is a non-zero easy box coefficient because $A_{4}\left(-l_{1}^{-h_{a}}, 2_{\bar{Q}}^{+}, 3_{Q}^{-}, l_{123}^{-h_{b}}\right)$ no longer vanishes for $h_{a}=h_{b}$. 


\begin{tabular}{|l|c|l|c|}
\hline \multicolumn{1}{|c|}{ Scalar integral } & Coefficient & \multicolumn{1}{|c|}{ Scalar integral } & Coefficient \\
\hline 1. $C_{0}\left(p_{23}, k_{4} ; 0,0,0\right)$ & $c_{23 \mid 4}$ & 6. $C_{0}\left(k_{1}, p_{23}, 0,0,0\right)$ & $c_{1 \mid 23}$ \\
2. $C_{0}\left(p_{12}, p_{3} ; 0, m, 0\right)$ & $c_{12 \mid 3}$ & 7. $C_{0}\left(p_{3}, k_{4}, m, 0,0\right)$ & $c_{3 \mid 4}$ \\
3. $C_{0}\left(p_{12}, p_{34} ; 0, m, 0\right)$ & $c_{12 \mid 34}$ & 8. $C_{0}\left(k_{1}, p_{2}, 0,0, m\right)$ & $c_{1 \mid 2}$ \\
4. $C_{0}\left(p_{2}, p_{3} ; 0, m, 0\right)$ & $c_{2 \mid 3}$ & $9 . C_{0}\left(k_{1}, p_{234}, 0,0,0\right)$ & $c_{1 \mid 234}$ \\
5. $C_{0}\left(p_{2}, p_{34} ; 0, m, 0\right)$ & $c_{2 \mid 34}$ & 10. $C_{0}\left(p_{123}, k_{4}, 0,0,0\right)$ & $c_{123 \mid 4}$ \\
\hline
\end{tabular}

Table 2. Scalar triangle integrals appearing in the leading colour amplitude $A_{6}^{\text {lc }}(1,2,3,4)$ and the notation used in the text to denote their coefficients.

\subsection{Calculation of triangle coefficients}

The leading colour amplitude receives contributions from the ten triangle scalar integrals listed in table 2 . The coefficients of Triangles 1-4 are calculated directly as detailed in the subsection below. Triangle 5 can be simply obtained by symmetry,

$$
c_{2 \mid 34}\left(h_{3}, h_{2}\right)=- \text { flip }\left[c_{12 \mid 3}\left(-h_{2},-h_{3}\right)\right] .
$$

The coefficients of the five remaining triangles are then uniquely determined by the known divergence structure [23] of the amplitude, eq. (4.1). The terms in eq. (4.1) proportional to $\log \left(-2 k_{1} \cdot p_{2}\right) / \epsilon$ and $\log \left(-2 k_{4} \cdot p_{3}\right) / \epsilon$ fix two of the triangle coefficients,

$$
\begin{aligned}
& c_{1 \mid 2}=-\left(\langle 1|\mathfrak{2}| 1]\left(-i A_{6}^{\text {tree }}\right)+\frac{d_{1|2| 3}}{s_{23}}+\frac{d_{1|2| 34}}{s_{234}}\right), \\
& c_{3 \mid 4}=-\left(\langle 4|\mathbf{3}| 4]\left(-i A_{6}^{\text {tree }}\right)+\frac{d_{2|3| 4}}{s_{23}}+\frac{d_{12|3| 4}}{s_{123}}\right),
\end{aligned}
$$

while the absence of single poles of the form $\log (s) / \epsilon$ for $s \in\left\{s_{23}, s_{1234}, s_{123}, s_{234}\right\}$ requires the following relations,

$$
\begin{aligned}
c_{1 \mid 23} & =\langle 1|2+3| 1]\left(\frac{d_{1|2| 3}}{s_{23}\langle 1|\mathbf{2}| 1]}+\frac{d_{2|3| 4}}{s_{23}\langle 4|\mathbf{3}| 4]}-\frac{c_{23 \mid 4}}{\langle 4|2+3| 4]}-\frac{2 d_{1|23| 4}}{\langle 1|2+3| 4|\langle 4|2+3| 1]}\right), \\
c_{1 \mid 234} & =\langle 1|2+3+4| 1]\left(\frac{d_{1|2| 3}}{s_{23}\langle 1|\mathbf{2}| 1]}+\frac{d_{1|2| 34}}{s_{234}\langle 1|\mathbf{2}| 1]}-\frac{c_{1 \mid 23}}{\langle 1|2+3| 1]}\right), \\
c_{123 \mid 4} & =\langle 4|1+2+3| 4]\left(\frac{d_{12|3| 4}}{s_{123}\langle 4|\mathbf{3}| 4]}+\frac{d_{1|2| 34}}{s_{234}\langle 1|\mathbf{2}| 1]}-\frac{c_{1 \mid 234}}{\langle 1|2+3+4| 1]}+\frac{2 d_{1|23| 4}}{\langle 1|2+3| 4]\langle 4|2+3| 1]}\right) .
\end{aligned}
$$

These expressions are written in terms of already-calculated box and triangle coefficients, the leading order amplitude, and the other triangle coefficients discussed below.

\subsubsection{Forde method for triangle coefficients}

We will calculate the coefficients of the triangle integrals using the method of Forde [13]. Triangles 2, 3 and 4 will require a slight extension of the formalism to include one of the internal propagators with a mass. We first review the case of three massless internal momenta, shown in figure 10 in order to introduce our notation which differs from that of Forde. 


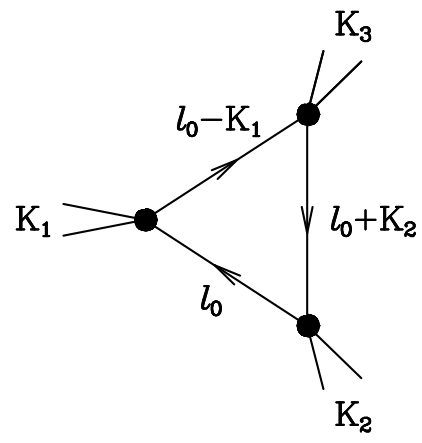

Figure 10. Triangle diagram showing the momentum parameterization. All momenta are outgoing, $K_{1}+K_{2}+K_{3}=0$.

Defining $l_{1}$ and $l_{2}$ as follows,

$$
l_{1}^{\mu}=l_{0}^{\mu}-K_{1}^{\mu}, \quad l_{2}^{\mu}=l_{0}^{\mu}+K_{2}^{\mu},
$$

the cut loop momenta $\left(l_{i}^{2}=0\right), i=0,1,2$ may be written in the following general form,

$$
l_{i}^{\mu}=x_{i} K_{1}^{b, \mu}+y_{i} K_{2}^{b, \mu}+\frac{t}{2}\left\langle K_{1}^{b}\left|\gamma^{\mu}\right| K_{2}^{b}\right]+\frac{x_{i} y_{i}}{2 t}\left\langle K_{2}^{b}\left|\gamma^{\mu}\right| K_{1}^{b}\right]
$$

All momenta can be expanded in terms of massless momenta, $K_{1}^{b}$ and $K_{2}^{b}$,

$$
\begin{aligned}
& K_{1}=K_{1}^{b}+\frac{S_{1}}{\gamma} K_{2}^{b}, \\
& K_{2}=K_{2}^{b}+\frac{S_{2}}{\gamma} K_{1}^{b}, \\
& K_{3}=-\left(1+\frac{S_{2}}{\gamma}\right) K_{1}^{b}-\left(1+\frac{S_{1}}{\gamma}\right) K_{2}^{b},
\end{aligned}
$$

where $S_{i}=K_{i}^{2}$ and $\gamma=\left\langle K_{1}^{b}\left|K_{2}^{b}\right| K_{1}^{b}\right]=2 K_{2}^{b} \cdot K_{1}^{b}$. The inverse relations are,

$$
K_{1}^{b, \mu}=\frac{K_{1}^{\mu}-\left(S_{1} / \gamma\right) K_{2}^{\mu}}{1-\left(S_{1} S_{2} / \gamma^{2}\right)}, \quad K_{2}^{b, \mu}=\frac{K_{2}^{\mu}-\left(S_{2} / \gamma\right) K_{1}^{\mu}}{1-\left(S_{1} S_{2} / \gamma^{2}\right)}
$$

The dot product of $K_{1}$ and $K_{2}$ in eq. (4.28) produces a quadratic equation for $\gamma$, the solutions of which express $\gamma$ in terms of the external momenta,

$$
\gamma_{ \pm}=\left(K_{1} \cdot K_{2}\right) \pm \sqrt{\Delta}, \quad \Delta=\left(K_{1} \cdot K_{2}\right)^{2}-S_{1} S_{2}
$$

From eq. (4.27) the massless vectors $l_{i}$ can be expressed as a linear combination of the spinor solutions for the vectors $K_{1}^{b}$ and $K_{2}^{b}$,

$$
\left\langle l_{i}\right|=t\left\langle K_{1}^{b}\right|+y_{i}\left\langle K_{2}^{b}\right|, \quad\left[l_{i} \mid=\frac{x_{i}}{t}\left[K_{1}^{b} \mid+\left[K_{2}^{b} \mid .\right.\right.\right.
$$


The on-shell conditions $l_{i}^{2}=0$ for $i=0,1,2$ allow us to derive the coefficients, $x_{i}$ and $y_{i}$,

$$
\begin{array}{ll}
y_{0}=\frac{S_{1}\left(\gamma+S_{2}\right)}{\left(\gamma^{2}-S_{1} S_{2}\right)}, & x_{0}=-\frac{S_{2}\left(\gamma+S_{1}\right)}{\left(\gamma^{2}-S_{1} S_{2}\right)}, \\
y_{1}=y_{0}-\frac{S_{1}}{\gamma}=\frac{S_{1} S_{2}\left(\gamma+S_{1}\right)}{\gamma\left(\gamma^{2}-S_{1} S_{2}\right)}, & x_{1}=x_{0}-1=-\frac{\gamma\left(\gamma+S_{2}\right)}{\gamma^{2}-S_{1} S_{2}}, \\
y_{2}=y_{0}+1=\frac{\gamma\left(\gamma+S_{1}\right)}{\gamma^{2}-S_{1} S_{2}}, & x_{2}=x_{0}+\frac{S_{2}}{\gamma}=-\frac{S_{1} S_{2}\left(\gamma+S_{2}\right)}{\gamma\left(\gamma^{2}-S_{1} S_{2}\right)} .
\end{array}
$$

The spinor products can be expressed as follows,

$$
\begin{aligned}
{\left[l l_{1}\right] } & =\frac{x_{1}-x_{0}}{t}\left[K_{2}^{b} K_{1}^{b}\right]=-\frac{1}{t}\left[K_{2}^{b} K_{1}^{b}\right], \\
\left\langle l l_{1}\right\rangle & =t\left(y_{1}-y_{0}\right)\left\langle K_{1}^{b} K_{2}^{b}\right\rangle=-\frac{t S_{1}}{\gamma}\left\langle K_{1}^{b} K_{2}^{b}\right\rangle, \\
{\left[l l_{2}\right] } & =\frac{x_{2}-x_{0}}{t}\left[K_{2}^{b} K_{1}^{b}\right]=\frac{S_{2}}{\gamma t}\left[K_{2}^{b} K_{1}^{b}\right], \\
\left\langle l l_{2}\right\rangle & =t\left(y_{2}-y_{0}\right)\left\langle K_{1}^{b} K_{2}^{b}\right\rangle=t\left\langle K_{1}^{b} K_{2}^{b}\right\rangle, \\
{\left[l_{1} l_{2}\right] } & =\frac{x_{2}-x_{1}}{t}\left[K_{2}^{b} K_{1}^{b}\right]=\frac{1}{t}\left(1+\frac{S_{2}}{\gamma}\right)\left[K_{2}^{b} K_{1}^{b}\right], \\
\left\langle l_{1} l_{2}\right\rangle & =t\left(y_{2}-y_{1}\right)\left\langle K_{1}^{b} K_{2}^{b}\right\rangle=t\left(1+\frac{S_{1}}{\gamma}\right)\left\langle K_{1}^{b} K_{2}^{b}\right\rangle .
\end{aligned}
$$

Turning now to the case where the propagator with momentum $l_{2}$ has a mass, we have that $l_{0}^{2}=l_{1}^{2}=0$ and $l_{2}^{2}=m^{2}$. Since $l_{2}$ is no longer massless it does not have an expansion of the form eq. (4.31). The results for the coefficients in the expansion defined in eq. (4.31) for $l_{0}$ and $l_{1}$ are,

$$
\begin{array}{ll}
y_{0}=\frac{S_{1}\left(\gamma+S_{2}-m^{2}\right)}{\left(\gamma^{2}-S_{1} S_{2}\right)}, & x_{0}=\frac{\gamma m^{2}-S_{2}\left(\gamma+S_{1}\right)}{\left(\gamma^{2}-S_{1} S_{2}\right)}, \\
y_{1}=y_{0}-\frac{S_{1}}{\gamma}=\frac{S_{1}\left(S_{2}\left(1+\left(S_{1} / \gamma\right)\right)-m^{2}\right)}{\gamma^{2}-S_{1} S_{2}}, & x_{1}=x_{0}-1=\frac{\gamma m^{2}-\gamma\left(S_{2}+\gamma\right)}{\gamma^{2}-S_{1} S_{2}} .
\end{array}
$$

With these results in hand we can then compute the coefficients for triangles $2-4$ with the loop momentum assignments as shown in figure 11 by making the replacements:

- Triangle 2: $K_{1}=-p_{123}, K_{2}=p_{3}$.

- Triangle 3: $K_{1}=-p_{1234}, K_{2}=p_{34}$.

- Triangle 4: $K_{1}=-p_{23}, K_{2}=p_{3}$.

For the case of triangles 2 and 4 , we see that the coefficients are particularly simple since $S_{2}=m^{2}$.

We shall sketch the calculation of the coefficient of triangle 4 because it is the simplest. We have $S_{1}=p_{23}^{2}=s_{23}$ and $S_{2}=p_{3}^{2}=m^{2}$ and hence see that,

$$
\gamma_{ \pm}=K_{1} \cdot K_{2} \pm \sqrt{\left(K_{1} \cdot K_{2}\right)^{2}-S_{1} S_{2}}=-\beta_{\mp} s_{23} \text {. }
$$



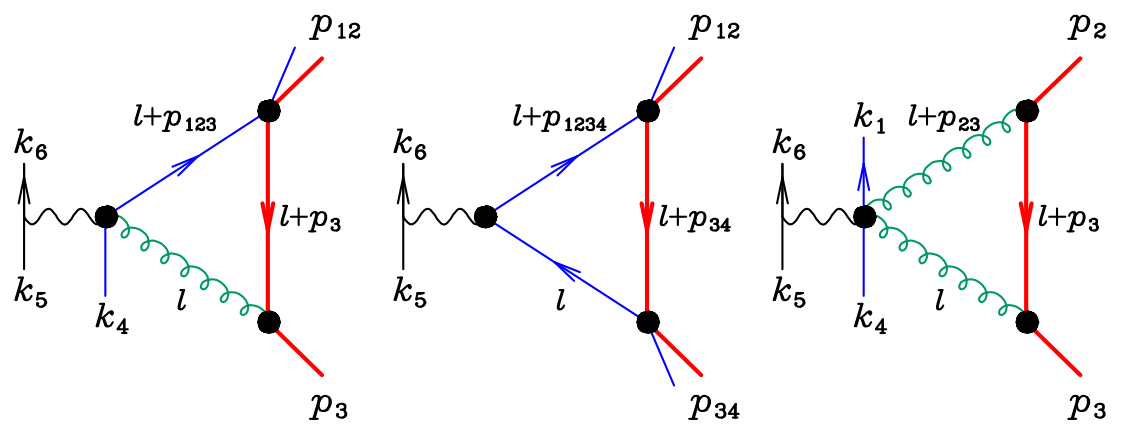

Figure 11. Diagrams for the calculation of the coefficients of the scalar integrals $C_{0}\left(p_{12}, p_{3} ; 0, m, 0\right)$, $C_{0}\left(p_{12}, p_{34} ; 0, m, 0\right)$ and $C_{0}\left(p_{2}, p_{3} ; 0, m, 0\right)$. The massive quark is represented by the heavy (red) line. The momenta on the external lines are all outgoing.

\begin{tabular}{|l|c|c|c|}
\hline \multicolumn{1}{|c|}{ Scalar integral } & Coefficient & Scalar integral & Coefficient \\
\hline 1. $B_{0}\left(p_{1234} ; 0,0\right)$ & $b_{1234}$ & $4 . B_{0}\left(p_{12} ; 0, m\right)$ & $b_{12}$ \\
2. $B_{0}\left(p_{23} ; 0,0\right)$ & $b_{23}$ & 5. $B_{0}\left(p_{34} ; 0, m\right)$ & $b_{34}$ \\
3. $B_{0}\left(p_{123} ; 0,0\right)$ & $b_{123}$ & $6 . B_{0}\left(p_{234} ; 0,0\right)$ & $b_{234}$ \\
\hline$B_{0}\left(p_{2} ; 0, m\right)$ & $b_{2}$ & $A_{0}(m)$ & $a$ \\
\hline
\end{tabular}

Table 3. Scalar bubble and tadpole integrals appearing in the leading colour amplitude $A_{6}^{\text {lc }}(1,2,3,4)$ and the notation used in the text to denote their coefficients.

The lightlike momenta, eq. (4.29), for the two solutions for $\gamma$ reduce to,

$$
\begin{aligned}
& K_{1}^{b}=-k_{2}, \quad K_{2}^{b}=\beta_{+} k_{3}, \quad \text { for } \gamma=\gamma_{-}=-\beta_{+} s_{23}, y_{0}=-\frac{1}{\beta}, x_{0}=-\frac{\beta_{-}}{\beta} \\
& K_{1}^{b}=-k_{3}, \quad K_{2}^{b}=\beta_{-} k_{2}, \quad \text { for } \gamma=\gamma_{+}=-\beta_{-} s_{23}, y_{0}=+\frac{1}{\beta}, x_{0}=+\frac{\beta_{+}}{\beta} .
\end{aligned}
$$

Using these assignments we find that the formulae for the coefficients of this triangle are relatively compact. For example, for the $\{-,+\}$ spin labels the result is,

$$
\begin{aligned}
c_{2 \mid 3}(-,+)= & \frac{1}{2 s_{56}}\left[\left\{\frac { [ 4 5 ] } { s _ { 1 2 3 } } \left(-\frac{\beta_{-}\langle 13\rangle^{2}\langle 6|(1+2)| 3]}{\langle 12\rangle}-\frac{\beta_{+}^{2}\langle 12\rangle[12]^{2}\langle 6|(1+2)| 3]}{\beta[13]^{2}}\right.\right.\right. \\
& +\frac{\beta_{+} \beta_{-}\langle 12\rangle[12]\langle 6|(1+3)| 2]}{\beta[13]}-\frac{\beta_{+} \beta_{-}\langle 13\rangle[12]\langle 6|(1+2)| 3]}{\beta[13]} \\
& \left.+\frac{\left(\beta^{2}-\beta_{-} \beta_{+} \beta+4 \beta_{-} \beta_{+}^{2}\right)}{\beta}\langle 13\rangle\langle 6|(1+3)| 2]\right)+\frac{\beta_{-}\langle 13\rangle\langle 16\rangle[45]}{\langle 12\rangle} \\
& \left.\left.-\frac{\beta_{+}^{2}\langle 23\rangle\langle 4|(2+3)| 5]\langle 6|(1+2)| 3]}{\beta\langle 24\rangle^{2}[13]}+\frac{\beta_{+}\langle 3|(2+4)| 5]\langle 6|(1+2)| 3]}{\langle 24\rangle[13]}\right\}-\{\text { flip }\}\right] .
\end{aligned}
$$

\subsection{Calculation of bubble and tadpole coefficients}

The bubble integrals present in the leading colour amplitude are shown in table 3 . The coefficients of bubbles $1-4$ are computed by using the method of spinor integration $[11,12$, 
20]. This is straightforward to apply for bubbles 1-3 but requires a small modification for bubble 4 due to the effect of the massive propagator. The modified method is described in the subsection below. Coefficients of the bubble integrals 5 and 6 are then obtained by symmetry,

$$
\begin{aligned}
b_{34}\left(h_{3}, h_{2}\right) & =- \text { flip }\left[b_{12}\left(-h_{2},-h_{3}\right)\right], \\
b_{234}\left(h_{3}, h_{2}\right) & =- \text { flip }\left[b_{123}\left(-h_{2},-h_{3}\right)\right] .
\end{aligned}
$$

All occurrences of $B_{0}\left(p_{3} ; 0, m\right)$ have been replaced by $B_{0}\left(p_{2} ; 0, m\right)$, so we only need to determine the coefficient $b_{2}$. A linear combination of the coefficient $b_{2}$ and $a$ is determined from the known form of the single pole in $\epsilon$, (i.e. the single pole with no associated logarithm in the expansion of eq. (4.1)),

$$
b_{2}+m^{2} a=\frac{8}{3}(-i) A_{6}^{\text {tree }}-b_{123}-b_{234}-b_{23}-b_{1234}-b_{12}-b_{34} .
$$

eq. (4.39) is sufficient to fix the poles and the logarithms, but since,

$$
\begin{aligned}
A_{0}(m) / m^{2} & =\left(\frac{\mu^{2}}{m^{2}}\right)^{\epsilon}\left(\frac{1}{\epsilon}+1\right), \\
B_{0}\left(p_{2} ; 0, m\right) & =\left(\frac{\mu^{2}}{m^{2}}\right)^{\epsilon}\left(\frac{1}{\epsilon}+2\right),
\end{aligned}
$$

it leaves the constant term undetermined. In order to separately fix the coefficients $a$ and $b_{2}$ we perform a direct Feynman diagram computation of the tadpole coefficient $a$ to find,

$$
\begin{aligned}
a(-,+) & =0, \\
a(+,-) & =0, \\
m^{2} a(-,-) & =\frac{1}{2}(-i) A_{6}^{\text {tree }}\left(1_{q}^{-}, 2_{\bar{Q}}^{-}, 3_{\bar{Q}}^{-}, 4_{\bar{q}}^{+}, 5_{\bar{\ell}}^{+}, 6_{\ell}^{-}\right), \\
m^{2} a(+,+) & =\frac{1}{2}(-i) A_{6}^{\text {tree }}\left(1_{q}^{-}, 2_{\bar{Q}}^{+}, 3_{Q}^{+}, 4_{\bar{q}}^{+}, 5_{\bar{\ell}}^{+}, 6_{\ell}^{-}\right) .
\end{aligned}
$$

These results for $a$ are valid in any covariant gauge.

\subsubsection{Bubble integral with one massive propagator}

To set up the formalism we consider the scalar bubble integral with one massive propagator, as shown in figure 12 (bubbles 4 and 5 are of this type). The evaluation of the coefficient of this bubble integral starts from the identity,

$$
\int d^{4} l \delta^{(+)}\left(l^{2}\right) \delta^{(+)}\left((l-P)^{2}-m^{2}\right)=\int_{0}^{\infty} t d t \int\langle l d l\rangle[l d l] \delta\left(P^{2}-m^{2}-t\langle l|P| l]\right),
$$

so that the formalism follows through as in the massless case, except that $t$ is now frozen at the value,

$$
t=\frac{P^{2}-m^{2}}{\langle l|P| l]}
$$




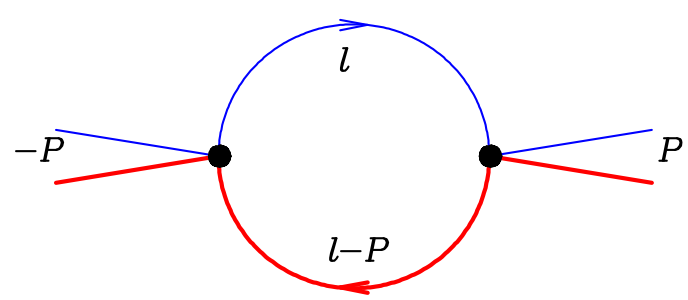

Figure 12. Diagram for the calculation of the coefficient of the scalar integral $B_{0}(P ; 0, m)$. The massive quark is represented by the heavy (red) line. The momenta on the external lines are all outgoing.

The discontinuity of the scalar integral is given by,

$$
\begin{aligned}
\Delta I_{2}(P, m) & =\int d^{4} l \delta^{(+)}\left(l^{2}\right) \delta^{(+)}\left((l-P)^{2}-m^{2}\right) \\
& =\int \frac{\langle l d l\rangle[l d l]}{\langle l|P| l]}\left(\frac{P^{2}-m^{2}}{\langle l|P| l]}\right)
\end{aligned}
$$

Using the standard formula $[11,20]$ we can express the integrand as a total derivative, and perform one of the spinor integrations,

$$
[l d l]\left(\frac{[\eta l]^{n}}{\langle l|P| l]^{n+2}}\right)=\left[d l \partial_{l}\right]\left(\frac{1}{n+1} \frac{1}{\langle l|P| \eta]} \frac{[\eta l]^{n+1}}{\langle l|P| l]^{n+1}}\right) .
$$

We obtain,

$$
\Delta I_{2}(P, m)=\left(P^{2}-m^{2}\right) \int \frac{\langle l d l\rangle[\eta l]}{\langle l|P| \eta]\langle l|P| l]},
$$

where $\eta$ is an arbitrary massless momentum. We perform the final integral over $\langle l d l\rangle$ by inspecting the residues of the pole in the integrand when $\langle l|=[\eta \mid P$ to find,

$$
\begin{aligned}
\Delta I_{2}(P, m) & =-\left(P^{2}-m^{2}\right) \frac{[\eta|P| \eta\rangle}{P^{2}\langle\eta|P| \eta]} \\
& =-\left(\frac{P^{2}-m^{2}}{P^{2}}\right) .
\end{aligned}
$$

So, in contrast to the massless case, when applying the spinor integration approach an additional rescaling factor of $P^{2} /\left(P^{2}-m^{2}\right)$ must be applied in order to obtain the coefficient of the scalar bubble integral.

\subsection{Calculation of the rational terms}

The purely rational terms are calculated using a traditional Feynman diagram approach, with tensor integrals handled using Passarino-Veltman reduction [26]. This might seem like a retrograde step, ineluctably leading to the algebraic complexity that we have been trying to avoid in this amplitude calculation. However two details of our particular case make it quite simple.

First, in the calculation of the rational part we only need to consider diagrams that violate the cut-constructibility condition [9]. This states that if $n$-point integrals $(n>2)$ 


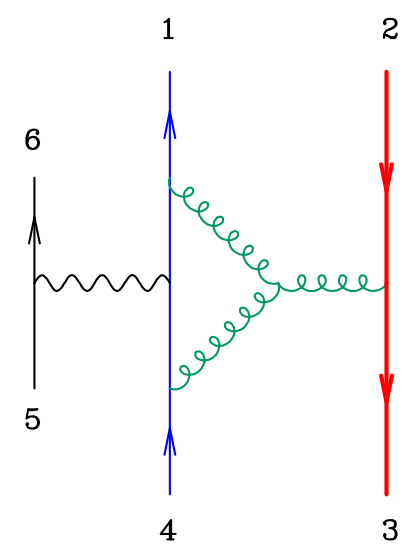

Figure 13. The box diagram contributing to the rational part in leading colour.

have at most $n-2$ powers of the loop momentum in the numerator of the integrand, and the two-point integrals have at most one power of the loop momentum, they will be cut-constructible. For our particular calculation the pentagon diagrams are all cutconstructible, and, in leading colour, there is only one box diagram, shown in figure 13, which is not cut-constructible and hence gives a rational part. The calculation of the rational parts from lower point diagrams, $n=3,2$ does not lead to great algebraic complexity. Second, the rational terms are generated by terms in the Passarino-Veltman decomposition that involve the metric tensor, $g^{\mu \nu}$. Therefore any terms that are not proportional to $g^{\mu \nu}$ may be discarded at an early stage of the computation.

The results for the rational terms turn out to be quite simple. For the $\{-,+\}$ case we find,

$$
\begin{aligned}
& R^{\mathrm{lc}}\left(1_{q}^{-}, 2_{\bar{Q}}^{+}, 3_{Q}^{-}, 4_{\bar{q}}^{+}, 5_{\bar{\ell}}^{+}, 6_{\ell}^{-}\right)= \\
& -\frac{2}{9}(-i) A_{6}^{\mathrm{tree}}\left(1_{q}^{-}, 2_{\bar{Q}}^{+}, 3_{Q}^{-}, 4_{\bar{q}}^{+}, 5_{\bar{\ell}}^{+}, 6_{\ell}^{-}\right) \\
& \quad+\left\{\frac { \langle 1 6 \rangle } { 2 s _ { 5 6 } } \left[\frac{\langle 13\rangle[15][12]}{\langle 1|(2+3)| 1]\langle 4|(2+3)| 1]}-\frac{\langle 13\rangle[15]}{s_{23}\langle 1|(2+3+4)| 1]}\left(\frac{s_{234}[12]}{\langle 4|(2+3)| 1]}+[24]\right)\right.\right. \\
& \left.\left.\quad+\frac{[24]}{s_{234}}\left(\frac{[25]}{[23]}-\frac{\langle 34\rangle[45]}{\langle 4|(2+3)| 4]}\right)\right]\right\}-\{\text { flip }\},
\end{aligned}
$$

where the flip operation is defined in eq. (3.9). The contribution for the opposite spin labels on the heavy quark line is simply related to this one,

$$
R^{\mathrm{lc}}\left(1_{q}^{-}, 2_{\bar{Q}}^{-}, 3_{Q}^{+}, 4_{\bar{q}}^{+}, 5_{\bar{\ell}}^{+}, 6_{\ell}^{-}\right)=R^{\mathrm{lc}}\left(1_{q}^{-}, 3_{\bar{Q}}^{+}, 2_{Q}^{-}, 4_{\bar{q}}^{+}, 5_{\bar{\ell}}^{+}, 6_{\ell}^{-}\right) .
$$

For the $\{-,-\}$ spin labels the result is,

$$
\begin{aligned}
R^{\mathrm{lc}}\left(1_{q}^{-}, 2_{\bar{Q}}^{-}, 3_{Q}^{-}, 4_{\bar{q}}^{+}, 5_{\bar{\ell}}^{+}, 6_{\ell}^{-}\right)=-\frac{2}{9}(-i) A_{6}^{\mathrm{tree}}\left(1_{\bar{q}}^{-}, 2_{\bar{Q}}^{-}, 3_{Q}^{-}, 4_{\bar{q}}^{+}, 5_{\bar{\ell}}^{+}, 6_{\ell}^{-}\right) \\
+\mathcal{N}_{--}\left(\left\{\frac { \langle 1 6 \rangle } { 2 s _ { 5 6 } } \left[\frac{[15]}{\langle 1|2+3+4| 1] s_{23}}\left(\frac{s_{234}(\langle 1|2| 1]-\langle 1|3| 1])}{\langle 4|2+3| 1]}-(\langle 1|2| 4]-\langle 1|3| 4])\right)\right.\right.\right. \\
\left.\left.\left.\quad-\frac{(\langle 1|2| 1]-\langle 1|3| 1])}{\langle 1|2+3| 1]}\left(\frac{[15]}{\langle 4|2+3| 1]}+\frac{[45]}{s_{23}}\right)\right]\right\}+\{\text { flip }\}\right),
\end{aligned}
$$


where the flip symmetry applies to all terms inside the curly brackets $\{\ldots\}$ but not the prefactor $\mathcal{N}_{--}$, defined in eq. (2.13). The final combination of spin labels is obtained by symmetry,

$$
R^{\mathrm{lc}}\left(1_{q}^{-}, 2_{\bar{Q}}^{+}, 3_{Q}^{+}, 4_{\bar{q}}^{+}, 5_{\bar{\ell}}^{+}, 6_{\ell}^{-}\right)=- \text {flip }\left(R^{\mathrm{lc}}\left(1_{q}^{-}, 2_{\bar{Q}}^{-}, 3_{Q}^{-}, 4_{\bar{q}}^{+}, 5_{\bar{\ell}}^{+}, 6_{\ell}^{-}\right)\right),
$$

which we note is equivalent to replacing $\mathcal{N}_{--}$by $\mathcal{N}_{++}$in eq. (4.51).

\section{The results for primitive amplitude $A_{6}^{\text {sl }}$}

The subleading colour primitive amplitude is shown in figure 4. Fortunately with our choice of massive spinors, eq. (2.9), the entire result for all spin labels of the massive quark line can be obtained from the one-loop results of Bern, Dixon and Kosower in eqs. $(12.10,12.11)$ of ref. [18], after some replacements and manipulation. The calculation reported in ref. [18] was for zero mass quarks and leptons with the following helicity assignments,

$$
A_{6}^{\mathrm{sl}}\left(1_{Q}^{+}, 2_{\bar{Q}}^{-}, 3_{q}^{+}, 4_{\bar{q}}^{-}, 5_{\bar{l}}^{-}, 6_{l}^{+}\right) .
$$

According to figure 2 our standard labelling of the graphs (for one of the amplitudes whose spin labelling corresponds to a non-zero amplitude in the massless case) is,

$$
A_{6}^{\mathrm{sl}}\left(1_{q}^{-}, 2_{\bar{Q}}^{+}, 3_{Q}^{-}, 4_{\bar{q}}^{+}, 5_{\bar{l}}^{+}, 6_{l}^{-}\right) .
$$

To establish the correspondence between our massive amplitudes and the massless ones of ref. [18], we first consider the tree graphs. In our notation the tree amplitudes are given by eqs. (3.8), (3.10). Note that we must perform the interchanges $(1 \leftrightarrow 3),\langle\rangle \leftrightarrow[])$, to compare with eq. (12.9) of ref. [18]. As a consequence of our eq. (2.11) the massive $\{-,+\}$ tree-level amplitude, eq. (3.8), calculated from diagrams of figure 2 and expressed in terms of the massless vectors, $k_{i}$, is identical to the massless result presented in eq. (12.9) of ref. [18] after performing the above interchange.

This same transformation, flipping the sign of all helicities and interchanging 1 and 3 , can be used at one-loop level to obtain the bulk of the results for the massive theory. This is true for the upper two diagrams of figure 4, because the massive quark enters only in the form of the heavy quark current see, eqs. (2.11), (2.12). The primitive amplitude shown in figure 4 can be split into divergent $\left(V^{\mathrm{sl}}\right)$ and finite $\left(F^{\mathrm{sl}}\right)$ pieces as follows

$$
A_{6}^{\mathrm{sl}}=\left[A_{6}^{\mathrm{tree}} V^{\mathrm{sl}}+i F^{\mathrm{sl}}\right] \text {, }
$$

After performing the interchanges to reduce it to our notation, the singular part of the one-loop amplitude as reported by Bern, Dixon and Kosower in ref. [18], eq. (12.10) is,

$$
V^{\text {sl }}(1,2,3,4)=V^{\text {box }}(1,2,3,4)+V^{\text {vertex }}(1,2,3,4)
$$

where the two contributions correspond to the upper and lower row of figure 4 in the massless theory,

$$
\begin{aligned}
V^{\text {box }}(1,2,3,4) & =\left[-\frac{1}{\epsilon^{2}}\left(\frac{\mu^{2}}{-s_{14}}\right)^{\epsilon}-\frac{3}{2 \epsilon}\left(\frac{\mu^{2}}{-s_{14}}\right)^{\epsilon}-4\right], \\
V^{\text {vertex }}(1,2,3,4) & =\left[-\frac{1}{\epsilon^{2}}\left(\frac{\mu^{2}}{-s_{23}}\right)^{\epsilon}-\frac{3}{2 \epsilon}\left(\frac{\mu^{2}}{-s_{23}}\right)^{\epsilon}-\frac{7}{2},\right]
\end{aligned}
$$


where $s_{i j}=2 k_{i} \cdot k_{j} . V^{\text {vertex }}$ in eq. (5.6) is the complete correction to external vertex for a massless line in the FDH scheme. For the massive case this must be replaced by the vertex correction for a massive line, i.e. the result for the lower two diagrams of figure 4 . The result is,

$$
\begin{aligned}
& V_{+-}^{\text {vertex }}=V_{-+}^{\text {vertex }}=\left(\frac{\mu^{2}}{m^{2}}\right)^{\epsilon}\left(\frac{1}{2 \epsilon}-\frac{1}{2 \beta}\left(1+\beta^{2}\right)\left[\frac{\ln x}{\epsilon}+G(x)\right]-\frac{3}{2} \beta \ln x+\frac{1}{2}\right), \\
& V_{++}^{\text {vertex }}=V_{--}^{\text {vertex }}=V_{-+}^{\text {vertex }}+\frac{1}{2} \beta \ln x,
\end{aligned}
$$

where $\beta$ and $\beta_{ \pm}$are given in eq. (2.6), $x=-\beta_{-} / \beta_{+}$, and,

$$
G(x)=-2 \operatorname{Li}_{2}(-x)-2 \ln x \ln (1+x)+\frac{1}{2} \ln ^{2}(x)-\frac{\pi^{2}}{6}, \quad \operatorname{Li}_{2}(x)=-\int_{0}^{x} \frac{d z}{z} \ln (1-z) .
$$

The self energy corrections on the external massive lines will be accounted for separately in association with the wave function renormalization. This concludes our description of the divergent parts and the lower two graphs of figure 4 .

We now turn to the finite parts of the massive primitive amplitudes shown in the upper part of figure 4. Because of eq. (2.11), the results for the $\{+,-\}$ and $\{-,+\}$ amplitudes from the upper row of figure 4 in the massive theory, expressed in terms of the lightlike momenta $k_{i}$, are given by the results in the massless theory.

The only remaining issue is whether we can also obtain the massive $\{-,-\}$ and $\{+,+\}$ amplitudes from the massless results. Note that in ref. [18] the finite parts of the massless amplitudes are written in terms of certain symmetry operations in order to make the amplitudes more compact. As a first step we write out the amplitudes explicitly. With this result in hand we want to address the issue of whether the results obtained with an external fermionic current, eq. (2.11) (i.e. the massless one-loop amplitude), can be used to obtain the results with the fermionic currents of eq. (2.12). Thus expressed in our notation, the massless amplitude for helicity choice $\left(2_{\bar{Q}}^{+}, 3_{Q}^{-}\right)$must contain one $\langle 3|$ and one $\left.\mid 2\right]$. All other dependence on $k_{2}$ or $k_{3}$ can only enter in the combination $k_{2}+k_{3}$ which can be eliminated by momentum conservation. After the amplitude has been recast in this form, we can obtain the required result by replacing the current of eq. (2.11) with the current of eq. (2.12).

An example may help to clarify the procedure. We shall consider a particular box which contributes to the one-loop amplitude,

$$
A_{6}^{\mathrm{sl}}\left(1_{q}^{-}, 2_{\bar{Q}}^{h_{2}}, 3_{Q}^{h_{3}}, 4_{\bar{q}}^{+}, 5_{\bar{\ell}}^{+}, 6_{\ell}^{-}\right)=d_{4|1| 23}\left(h_{3}, h_{2}\right) D_{0}\left(k_{4}, k_{1}, p_{23} ; 0,0,0,0\right)+\ldots
$$

The result for this box coefficient in the massless helicity-conserving amplitude, (adapted from the first line of eq. (12.11) of ref. [18]) is,

$$
d_{4|1| 23}(-,+)=s_{14}\left(\frac{\langle 13\rangle^{2}[45]^{2}}{\langle 32\rangle[56][4|(2+3)| 1\rangle}-\frac{[1|(2+3)| 6\rangle^{2}[2|(1+3)| 4\rangle^{2}}{[32]\langle 56\rangle[1|(2+3)| 4\rangle^{3}}\right) .
$$




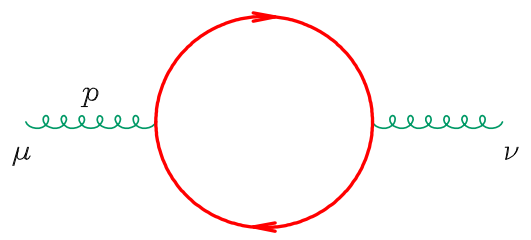

Figure 14. Fermion loop contribution to the gluon vacuum polarization.

This can be rewritten in a form which makes the $|3\rangle, \mid 2]$ and $k_{2}+k_{3}$ structure manifest,

$$
\begin{aligned}
& d_{4|1| 23}(-,+)=-s_{14} \\
& \quad \times\left(\frac{\langle 13\rangle\langle 1|(2+3)| 2][45]^{2}}{s_{23}[56][4|(2+3)| 1\rangle}-\frac{[1|(2+3)| 6\rangle^{2}\langle 3|(2+3)(1+2+3)| 4\rangle[2|(1+2+3)| 4\rangle}{s_{23}\langle 56\rangle[1|(2+3)| 4\rangle^{3}}\right) .
\end{aligned}
$$

Replacing the $\{-,+\}$-current, eq. (2.15), with the $\{-,-\}$-current, eq. (2.16), we obtain the result for the coefficient of this box in the massive amplitude labelled $\{-,-\}$

$$
\begin{aligned}
d_{4|1| 23}(-,-) & =-\mathcal{N}_{--} s_{14} \\
\times & \left(\frac{\langle 12\rangle\langle 13\rangle[45]^{2}}{\langle 32\rangle[56][4|(2+3)| 1\rangle}+\frac{[1|(2+3)| 6\rangle^{2}[2|(1+3)| 4\rangle[3|(1+2)| 4\rangle}{[32]\langle 56\rangle[1|(2+3)| 4\rangle^{3}}\right) .
\end{aligned}
$$

Carrying out this operation for all the terms in eq. (12.11) of ref. [18] we obtain the complete massive amplitude for the $\{-,-\}$ and $\{+,+\}$ spin labellings.

\section{Results for the primitive amplitudes $A_{6}^{l f}$ and $A_{6}^{h f}$}

The unrenormalized contribution of the fermion loop diagram, shown in figure 14, for a quark of mass $m$ is,

$$
\Pi^{\mu \nu}=i g^{2} c_{\Gamma}\left[g^{\mu \nu} p^{2}-p^{\mu} p^{\nu}\right] \Pi\left(p^{2}, m^{2}\right),
$$

where (not including the minus sign for a fermion loop),

$$
\Pi\left(p^{2}, m^{2}\right)=2 T_{R} \frac{1}{\epsilon}\left(\frac{\mu^{2}}{m^{2}}\right)^{\epsilon} \int_{0}^{1} d x \frac{4 x(1-x)}{\left[1-x(1-x) \frac{p^{2}}{m^{2}}\right]^{\epsilon}},
$$

and $T_{R}=\frac{1}{2}$. For $n_{l f}$ quarks which can be considered massless this becomes,

$$
\Pi\left(p^{2}, 0\right)=n_{l f}\left[\frac{2}{3} \frac{1}{\epsilon}\left(\frac{\mu^{2}}{-p^{2}}\right)^{\epsilon}+\frac{10}{9}\right]+O(\epsilon) .
$$

Thus in our notation, (c.f. eq. (3.13)) the result for the unrenormalized fermion-loop primitive is,

$$
A_{6}^{l f}\left(1_{q}^{+}, 2_{\bar{Q}}^{ \pm}, 3_{Q}^{\mp}, 4_{\bar{q}} ; 5_{\bar{\ell}}^{+}, 6_{\ell}^{-}\right)=A_{6}^{\mathrm{tree}}\left(1_{q}^{+}, 2_{\bar{Q}}^{ \pm}, 3_{Q}^{\mp}, 4_{\bar{q}} ; 5_{\bar{\ell}}^{+}, 6_{\ell}^{-}\right)\left[\frac{2}{3} \frac{1}{\epsilon}\left(\frac{\mu^{2}}{-s_{23}}\right)^{\epsilon}+\frac{10}{9}\right],
$$

where $A_{6}^{\text {tree }}$ are given in eqs. (3.8), (3.10). 


\section{Renormalization}

The amplitudes presented so far are bare amplitudes, which require ultraviolet renormalization. The renormalization scheme is slightly more complicated in the presence of massive particles [30] so we specify it in detail here. The requirements for our renormalization scheme are,

- The decoupling of heavy quarks should be manifest.

- The evolution equations for the running coupling and for the parton distribution functions should be the same as the equations in the theory without the heavy quark. Both the strong coupling and the parton distribution functions should run with the coefficients appropriate for the $\overline{\mathrm{MS}}$ scheme in the absence of the massive particles.

- The mass parameter should correspond to a pole mass.

These three requirements completely specify the renormalization scheme. If the diagram in question contains no heavy internal loops of heavy particles we use the $\overline{\mathrm{MS}}$ scheme. If on the other hand the diagram contains heavy loops we will perform subtraction at zero momentum, $p=0$. The resultant renormalized Green's function will be a function of $p^{2} / \mathrm{m}^{2}$ and hence exhibit decoupling as the mass of the heavy quarks becomes large.

The full renormalized amplitude, $A_{6 ; 1}^{R}$ is obtained by adding an overall counterterm,

$$
\begin{aligned}
& N_{c} A_{6 ; 1}^{R}=N_{c} A_{6 ; 1} \\
& \quad+g^{2} c_{\Gamma}\left\{-2\left(\frac{11}{6} N_{c}-\frac{n_{\mathrm{lf}}}{3}\right) \frac{1}{\epsilon}+\frac{2 n_{\mathrm{hf}}}{3}\left(\frac{1}{\epsilon}+\ln \frac{\mu^{2}}{m^{2}}\right)+\frac{N_{c}}{3}-C_{F}\left(\frac{3}{\epsilon}+3 \ln \frac{\mu^{2}}{m^{2}}+5\right)\right\} A_{6}^{\text {tree }} .
\end{aligned}
$$

We will now justify the contributions in the counterterm term by term. The first term in eq. (7.1) is the normal $\overline{M S}$ renormalization of the coupling constant, which includes the renormalization of the $n_{\mathrm{lf}}$ loops of massless fermions. The second term in eq. (7.1) deals with the case where we have $n_{\mathrm{hf}}$ heavy fermions. Renormalizing eq. (6.2) at $p^{2}=0$ to ensure decoupling of the heavy fermions as $m$ becomes large, we obtain for each of the $n_{\mathrm{hf}}$ heavy fermions,

$$
\Pi^{R}\left(p^{2}, m^{2}\right)=-4 \int_{0}^{1} d x x(1-x) \ln \left(1-x(1-x) \frac{p^{2}}{m^{2}}\right) .
$$

In the high mass limit $\Pi^{R}$ simplifies to,

$$
\Pi^{R}\left(s, m^{2}\right) \rightarrow \frac{2}{15} \frac{s}{m^{2}}+O\left(\frac{s^{2}}{m^{4}}\right) .
$$

Thus after renormalization the contribution of the heavy quark is given by (c.f. eq. (3.13))

$$
A_{6}^{h f}\left(1_{q}^{+}, 2_{\bar{Q}}^{ \pm}, 3_{Q}^{\mp}, 4_{\bar{q}} ; 5_{\bar{\ell}}^{+}, 6_{\ell}^{-}\right)=A_{6}^{\text {tree }}\left(1_{q}^{+}, 2_{\bar{Q}}^{ \pm}, 3_{Q}^{\mp}, 4_{\bar{q}} ; 5_{\bar{\ell}}^{+}, 6_{\ell}^{-}\right) \Pi^{R}\left(s_{23}, m^{2}\right) .
$$


We must also perform a finite renormalization of the gauge coupling [28] to translate from the FDH coupling to the normal $\overline{\mathrm{MS}}$ coupling,

$$
\alpha_{s}^{\mathrm{FDH}}=\alpha_{s}^{\overline{\mathrm{MS}}}\left(1+\frac{N_{c}}{6} \frac{\alpha_{s}^{\overline{\mathrm{MS}}}}{2 \pi}\right) .
$$

This explains the third term in eq. (7.1). The last term in eq. (7.1) represents the wave function renormalization for the two external massive fermions, calculated in appendix D. In the FDH scheme we have from eq. (D.10),

$$
Z_{Q}=1-g^{2} c_{\Gamma} C_{F}\left[\frac{3}{\epsilon}+3 \ln \left(\frac{\mu^{2}}{m^{2}}\right)+5\right]+O\left(g^{4}, \epsilon\right),
$$

independent of the gauge-fixing parameter in any covariant gauge.

Since our calculation is performed in the four-dimensional helicity scheme there is a further finite renormalization [28] required to arrive at the 't Hooft-Veltman scheme. We shall work consistently in the FDH scheme, so this will not be required.

\section{Implementation into MCFM}

The one-loop matrix elements, computed using the methods described above, have been included in a full next-to-leading order calculation of the $W Q \bar{Q}$ process. The amplitude for the lowest order process is given in eqs. (3.8), (3.10). In order to complete the NLO calculation the Born level amplitude and the one-loop amplitude must be supplemented with results for the real radiation diagrams and a method for cancelling infrared singularities between the two contributions. The tree-level real radiation process has been computed using the diagrams shown in figure 15, adopting the same choice of massive spinors as used in the virtual contribution. Infrared singularities are handled using the subtraction method [33] implemented using the dipole formulation [34] and extended to the case of massive emitters and spectators [35]. The full calculation will be made available as part of the the MCFM code [31, 32].

To provide a point of comparison we have timed the evaluation of the complete oneloop contribution, i.e. the interference in eq. (3.7), summed over all four possible spin labels on the heavy quark line. Using a standard $2.66 \mathrm{GHz}$ machine and compiling the code with the -02 flag of gfortran this operation takes $4 \mathrm{~ms}$ for a single phase space point, which is about a factor of sixty slower than evaluating the corresponding massless amplitudes as implemented in MCFM.

\subsection{Checks on the calculation}

A number of checks have been performed at various stages of the calculation.

1. At the level of the one-loop amplitude, we have cross-checked our calculation with a numerical implementation of generalized $d$-dimensional unitarity [36]. This check confirms the coefficients of all the scalar integrals and rational terms, as well as the complete (unrenormalized) amplitude, at any given phase space point. The values of the unrenormalized one-loop amplitudes at a specific phase space point are given in appendix E. These numbers may be useful in performing a check of our calculation. 

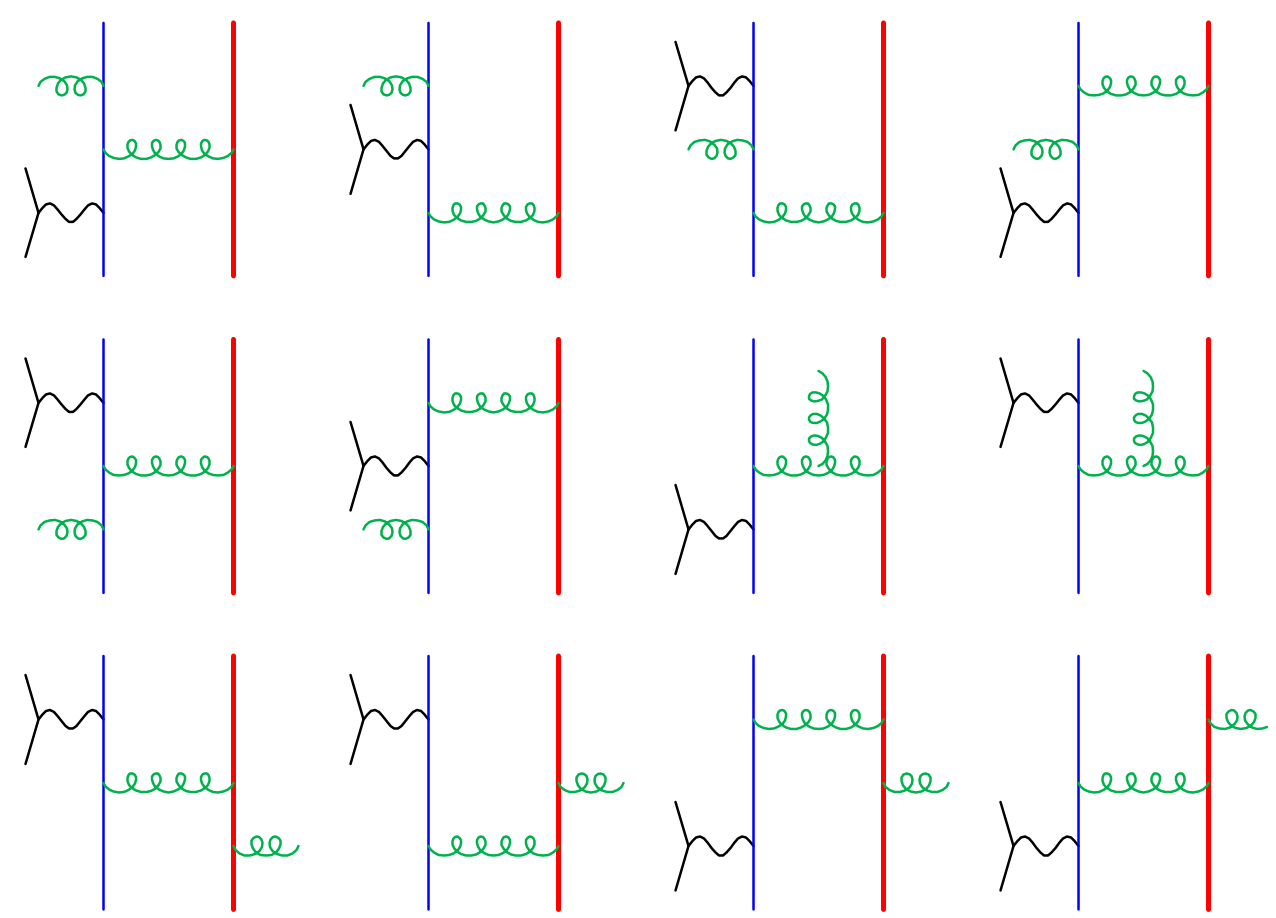

Figure 15. Feynman graphs appearing in the calculation of the real radiation amplitudes.

$\left.\begin{array}{|ll|}\hline m_{W}=80.44 \mathrm{GeV} & m_{b}=4.62 \mathrm{GeV} \\
m_{t}=172.6 \mathrm{GeV} & \sin ^{2} \theta_{w}=0.223 \\
G_{F}=1.16639 \cdot 10^{-5} \mathrm{GeV}^{-2} & \alpha=\frac{\sqrt{2}}{\pi} G_{F} m_{W}^{2} \sin ^{2} \theta_{w} \\
V_{u d}=V_{c s}=0.974 \mathrm{GeV} & V_{u s}=V_{c d}=0.227 \\
\text { PDF set: CTEQ6L1 } & \text { PDF set: CTEQ6M } \\
\alpha_{s}\left(m_{W}+2 m_{b}\right)=0.130345\end{array}\right\}$ LO $\quad$\begin{tabular}{l}
$\alpha_{s}\left(m_{W}+2 m_{b}\right)=0.118298$ \\
\hline
\end{tabular}

Table 4. Inputs used for the results presented in section 8.

2. The cancellation of infrared singularities is performed using a slight extension of the original dipole formulation in which the extent of each subtraction region is controlled by an additional parameter $[37,38]$. This parameter also appears in the integrated form of the dipole counterterms in such a way that the sum of real and virtual radiation does not depend upon its value. We have checked that this independence is indeed manifest in our calculation.

3. We have checked that our final results for the $W b \bar{b}$ integrated cross section agree with the values reported in the earlier calculations of refs. [2-4].

\subsection{Phenomenology}

For now we present only a limited set of results, focussing mainly on the comparison with previous calculations and presenting a simple distribution involving the decay products of the $W$ boson. We leave a detailed phenomenological study for a future work. 


\begin{tabular}{|cl|c|c|c|}
\hline \multicolumn{2}{|c|}{$\sqrt{s}$} & $7 \mathrm{TeV}$ & $8 \mathrm{TeV}$ & $14 \mathrm{TeV}$ \\
\hline \multirow{2}{*}{$W^{+} b \bar{b}$} & LO & $4.456(2)$ & $5.157(2)$ & $9.041(3)$ \\
& NLO & $8.655(9)$ & $10.58(2)$ & $23.51(3)$ \\
\hline \multirow{2}{*}{$W^{-} b \bar{b}$} & LO & $2.588(2)$ & $3.109(1)$ & $6.256(2)$ \\
& NLO & $5.053(5)$ & $6.353(6)$ & $15.55(2)$ \\
\hline
\end{tabular}

Table 5. LO and NLO cross sections (in picobarns) for $W b \bar{b}$ production at various energies of the LHC. Integration errors are shown in parentheses.

For convenience, we choose the same set of input parameters here as reported in ref. [3], which are summarized in table 4 . The final state is defined by the following cuts on the $b$-jets,

$$
p_{T}^{b}>25 \mathrm{GeV}, \quad\left|\eta^{b}\right|<2.5,
$$

where the jets are identified using the $k_{T}$ clustering algorithm with pseudo-cone size $R=0.7$. The results presented here are inclusive of the additional jet that may be present at NLO.

As already discussed previously, although we treat the $b$-quark as a massive particle when it appears in the final state, we use $n_{\mathrm{lf}}=5$ light flavours in the running of $\alpha_{s}$ and the PDF evolution. This is primarily for comparison with previous work [3]. We note that because of the smallness of $V_{c b}$ and $V_{u b}$ the $b$-quark distributions in the initial state make a negligible contribution. However because $s_{23}>4 m_{b}^{2}$ it is more appropriate to have a strong coupling constant running with $n_{\mathrm{lf}}=5$ active flavours.

We first present the cross sections for this process at the LHC, for center-of-mass energies $\sqrt{s}=7,8$ and $14 \mathrm{TeV}$. The results at LO and NLO are shown in table 5 where we have used a scale choice $\mu_{R}=\mu_{F}=m_{W}+2 m_{b}$ throughout, again to facilitate comparison with ref. [3]. For these results no cuts are applied on the decay products of the $W$ boson and the corresponding branching ratio is removed, so that the reported cross sections are for a $W$ that does not decay. In this way, one sees from the final column $(\sqrt{s}=14 \mathrm{TeV})$ that our results agree with those reported in ref. [3] at the level of $0.5 \%$. As noted in earlier work [39], at LHC energies the NLO corrections are substantial because of the influence of the quark-gluon initial state.

To illustrate a new quantity that may now be computed at NLO accuracy, in figure 16 we show the LO and NLO predictions for the quantity $R_{l j}^{\min }$ which is defined as the separation between the charged lepton and the closest jet,

$$
R_{l j}^{\min }=\min _{\{\text {jets }\}} \sqrt{\left(\eta_{\text {lepton }}-\eta_{\text {jet }}\right)^{2}+\left(\phi_{\text {lepton }}-\phi_{\text {jet }}\right)^{2}}
$$

In this equation the azimuthal angles and pseudorapidities (in the lab-frame) of the lepton and jets are denoted by $\phi$ and $\eta$ respectively. We see that the effect of the NLO corrections on the shape of this distribution is relatively minor, visible only for $R_{j l}^{\min }>1.5$. 


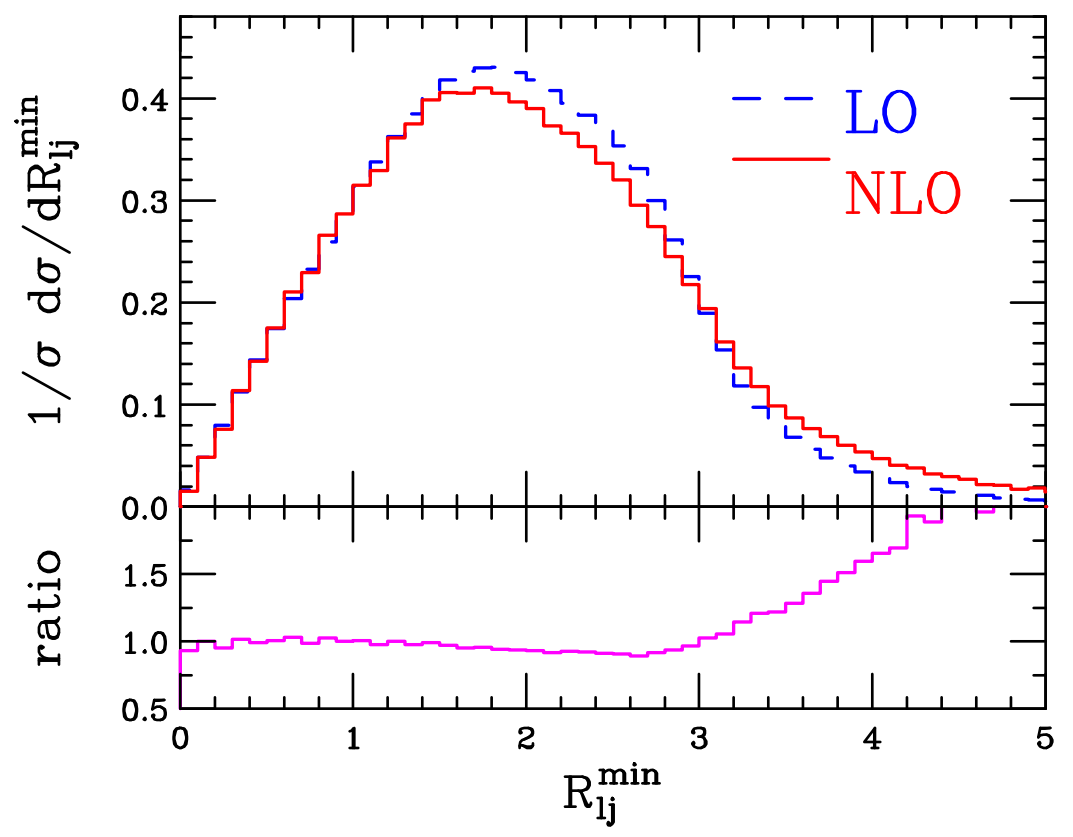

Figure 16. Distribution of the minimum separation between the electron and any jet, $R_{l j}$, defined in eq. (8.2) of the text, for $W^{-} b \bar{b}$ production at the $14 \mathrm{TeV}$ LHC. The distributions are normalized to unit area. The ratio of NLO to LO is shown in the lower part of the figure.

\section{Conclusions}

We have presented the first computation of the $W b \bar{b}$ cross section with massive $b$-quarks, including the lepton correlations present in the decay of the $W$-boson. This calculation required knowledge of the one-loop virtual corrections to the $q \bar{Q} Q \bar{q} \bar{\ell} \ell$ process retaining the mass for the heavy quarks $Q$. The one-loop amplitude was obtained using the spinor helicity formalism. This method has been extensively used for one-loop calculations with massless quarks, but rarely with massive quarks. The calculation required a number of modifications of standard techniques to cope with the presence of the mass.

Although our results are analytic we have not yet simplified them sufficiently to publish them in a journal article. Our results for the one-loop amplitudes will be included in the released version of MCFM, which is an appropriate method of publishing such results. Our analytic results did lead to a code which is fast and numerically stable. Using this code we intend to study the detailed phenomenology of this process in a future publication.

\section{Acknowledgments}

We are happy to acknowledge useful discussions with Fabrizio Caola and Kirill Melnikov. The work of SB has been supported in part by Danish Natural Science Research Council grant 10-084954. Fermilab is operated by Fermi Research Alliance, LLC, under contract DE-AC02-07CH11359 with the United States Department of Energy. 


\section{A Notation for spinor products}

The spinor notation is almost standard, but because of the presence of massive particles, it is important to make it explicit. We adopt the following notation for massless spinors,

$$
\begin{aligned}
& \left.|i\rangle=|i+\rangle=u_{+}\left(k_{i}\right), \mid i\right]=|i-\rangle=u_{-}\left(k_{i}\right), \\
& \langle i|=\langle i-|=\bar{u}_{-}\left(k_{i}\right),\left[i \mid=\langle i+|=\bar{u}_{+}\left(k_{i}\right) .\right.
\end{aligned}
$$

Further the spinor products are defined as,

$$
\begin{aligned}
\langle i j\rangle & =\langle i-\mid j+\rangle=\bar{u}_{-}\left(k_{i}\right) u_{+}\left(k_{j}\right), \\
{[i j] } & =\langle i+\mid j-\rangle=\bar{u}_{+}\left(k_{i}\right) u_{-}\left(k_{j}\right),
\end{aligned}
$$

with $k_{i}, k_{j}$ massless particles. With our convention,

$$
\langle i j\rangle[j i]=2 k_{i} \cdot k_{j}=s_{i j} .
$$

We also define the spinor strings.

$$
\begin{aligned}
\langle i|j| k] & \equiv\left\langle k_{i}-\left|\not k_{j}\right| k_{k}-\right\rangle, \\
\langle i|j+k| l] & \equiv\left\langle k_{i}-\left|\left(\not k_{j}+\not k_{k}\right)\right| k_{l}-\right\rangle, \\
\langle i|j k| l\rangle & \equiv\left\langle k_{i}-\left|\not k_{j} \not k_{k}\right| k_{l}+\right\rangle .
\end{aligned}
$$

For the case of a massless momentum $k_{j}$ we may write,

$$
\langle i|j| k]=\langle i j\rangle[j k],
$$

but for the case of a massive momentum, (in our notation the momenta $p_{2}$ and $p_{3}$ ), this separation in no longer possible. As a compact notation we therefore write in this case $\langle i|\mathbf{j}| k]$, denoting the massive momentum by a bold-face symbol.

The Schouten identity,

$$
[i k][m n]=[i n][m k]+[i m][k n],
$$

may be applied to these compound quantities. Thus we have,

$$
\langle i|\mathbf{j}| k][m n]=\langle i|\mathbf{j}| n][m k]+\langle i|\mathbf{j}| m][k n] .
$$

\section{B Tree level results}

\section{B.1 Results for $A\left(1_{g}, 2_{\bar{Q}}, 3_{Q}, 4_{g}\right)$}

As an example of the use of the massive spinors employed in the calculation we consider the tree-level two-quark two-gluon amplitude, $A\left(1_{g}, 2_{\bar{Q}}, 3_{Q}, 4_{g}\right)$. The fermions, with momenta $p_{2}$ and $p_{3}$ have a common mass $m$. The momenta are all outgoing so that $k_{1}+p_{2}+p_{3}+k_{4}=$ 0 . The amplitude is obtained by summing the contributions of the three Feynman diagrams, 


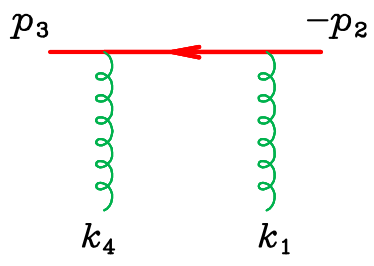

(a)

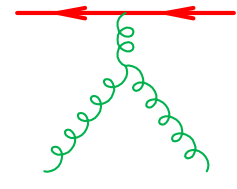

(b)

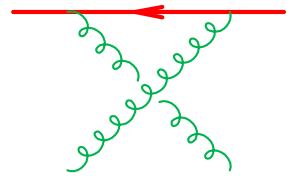

(c)

Figure 17. Diagrams for two gluons coupling to a massive quark line.

$M^{(a)}, M^{(b)}$ and $M^{(c)}$ shown in figure 17,

$$
A_{4}^{\text {tree }}=-\frac{i}{2} g^{2}\left[\left(T^{C_{4}} T^{C_{1}}\right)_{i_{3} i_{2}}\left(M^{(a)}+M^{(b)}\right)+\left(T^{C_{1}} T^{C_{4}}\right)_{i_{3} i_{2}}\left(M^{(c)}-M^{(b)}\right)\right],
$$

Normalizing the colour matrices as follows,

$$
\operatorname{Tr} T^{A} T^{B}=\delta^{A B},
$$

an explicit calculation yields the following contributions,

$$
\begin{aligned}
M^{(a)} & =\bar{u}\left(p_{3}\right) \phi_{4} \frac{\not p_{34}+m}{p_{34}^{2}-m^{2}} \phi_{1} v\left(p_{2}\right), \\
M^{(b)} & =\frac{\epsilon_{1} \cdot \epsilon_{4}}{k_{1} \cdot k_{4}} \bar{u}\left(p_{3}\right) \not \not_{1} v\left(p_{2}\right)-\frac{k_{1} \cdot \epsilon_{4}}{k_{1} \cdot k_{4}} \bar{u}\left(p_{3}\right) \phi_{1} v\left(p_{2}\right)+\frac{k_{4} \cdot \epsilon_{1}}{k_{1} \cdot k_{4}} \bar{u}\left(p_{3}\right) \phi_{4} v\left(p_{2}\right), \\
M^{(c)} & =\bar{u}\left(p_{3}\right) \phi_{1} \frac{\not p_{13}+m}{p_{13}^{2}-m^{2}} \phi_{4} v\left(p_{2}\right),
\end{aligned}
$$

where $p_{13}=k_{1}+p_{3}$ and $p_{34}=p_{3}+k_{4}$. From eq. (2.9) we write the massive spinors as,

$$
\begin{aligned}
& \bar{u}\left(p_{3}\right)=N_{3} \bar{u}\left(k_{2}\right)\left(\not p_{3}+m\right), \\
& v\left(p_{2}\right)=N_{2}\left(\not p_{2}-m\right) v\left(k_{3}\right),
\end{aligned}
$$

and then reorganize the calculation by using the identities [40],

$$
\begin{aligned}
& \left(\not \not_{3}+m\right) \not_{4} \equiv\left(\not \not_{34}-\not \not_{4}+m\right) \phi_{4} \\
& \equiv\left(2 p_{34}-k_{4}\right) \cdot \epsilon_{4}-\frac{1}{2}\left[\not \not_{4}, \phi_{4}\right]-\phi_{4}\left(\not p_{34}-m\right), \\
& \left(-\not \not_{12}+m\right) \phi_{1} \equiv-\left(2 p_{2}+k_{1}\right) \cdot \epsilon_{1}-\frac{1}{2}\left[\not k_{1}, \phi_{1}\right]+\phi_{1}\left(\not p_{2}+m\right) \text {. }
\end{aligned}
$$

Inserting these identities we may write new forms for $M^{(a)}$ and $M^{(c)}$,

$$
\begin{aligned}
M^{(i)} & =N_{2} N_{3} \bar{u}\left(k_{2}\right) \Gamma^{(i)}\left(\not p_{2}-m\right) v\left(k_{3}\right), \quad i=(a, b, c) \\
\Gamma^{(a)} & =\left\{\left[2 p_{3} \cdot \epsilon_{4}-\frac{1}{2}\left[\not k_{4}, \phi_{4}\right]\right] \frac{1}{2 p_{3} \cdot k_{4}}\left[-2 p_{2} \cdot \epsilon_{1}-\frac{1}{2}\left[\not k_{1}, \phi_{1}\right]\right]-\phi_{4} \phi_{1}\right\}, \\
\Gamma^{(c)} & =\left\{\left[2 p_{3} \cdot \epsilon_{1}-\frac{1}{2}\left[\not \not_{1}, \phi_{1}\right]\right] \frac{1}{2 p_{3} \cdot k_{1}}\left[-2 p_{2} \cdot \epsilon_{4}-\frac{1}{2}\left[\not k_{4}, \phi_{4}\right]\right]-\phi_{1} \phi_{4}\right\} .
\end{aligned}
$$


These formulae have the advantage that the $m$ dependence is corralled in a single place, in eq. (B.10). Moreover the overall form is simple and the last term in eqs. (B.11), (B.12) does not contain the massive propagator. $\Gamma^{(b)}$ can be read off from eq. (B.4). Inserting the appropriate polarization vectors we find,

$$
\begin{aligned}
& -i A\left(1_{g}^{-}, 2_{\bar{Q}}^{+}, 3_{Q}^{-}, 4_{g}^{+}\right)=-\frac{[24]^{2}\langle 1|\mathbf{2}| 4]}{[14][23]\langle 4|\mathbf{3}| 4]} \\
& -i A\left(1_{g}^{-}, 2_{\bar{Q}}^{-}, 3_{Q}^{+}, 4_{g}^{+}\right)=-\frac{\langle 12\rangle^{2}\langle 1|\boldsymbol{3}| 4]}{\langle 14\rangle\langle 23\rangle\langle 4|\mathbf{3}| 4]}, \\
& -i A\left(1_{g}^{-}, 2_{\bar{Q}}^{-}, 3_{Q}^{-}, 4_{g}^{+}\right)=2\left(\beta_{+}-\beta_{-}\right) \frac{m}{[23]} \frac{\langle 13\rangle^{2}[34]^{2}}{\langle 14\rangle[14]\langle 4|\mathbf{3}| 4]} \\
& -i A\left(1_{g}^{-}, 2_{\bar{Q}}^{+}, 3_{Q}^{+}, 4_{g}^{+}\right)=2\left(\beta_{+}-\beta_{-}\right) \frac{m}{\langle 23\rangle} \frac{\langle 13\rangle^{2}[34]^{2}}{\langle 14\rangle[14]\langle 4|\mathbf{3}| 4]} \\
& -i A\left(1_{g}^{-}, 2_{\bar{Q}}^{+}, 3_{Q}^{-}, 4_{g}^{-}\right)=0 \\
& -i A\left(1_{g}^{-}, 2_{\bar{Q}}^{-}, 3_{Q}^{+}, 4_{g}^{-}\right)=0, \\
& -i A\left(1_{g}^{-}, 2_{\bar{Q}}^{+}, 3_{Q}^{+}, 4_{g}^{-}\right)=-\frac{m \beta_{+}}{\langle 23\rangle} \frac{\langle 14\rangle^{2}}{\langle 4|\mathbf{3}| 4]} \\
& -i A\left(1_{g}^{-}, 2_{\bar{Q}}^{-}, 3_{Q}^{-}, 4_{g}^{-}\right)=\frac{m \beta_{-}}{[23]} \frac{\langle 14\rangle^{2}}{\langle 4|\mathbf{3}| 4]}
\end{aligned}
$$

B.2 Results for $A\left(1_{q}, 2_{\bar{q}}, 3_{g}\right)$

The tree-level results for the simple $q \bar{q} g$ amplitudes, stripped of overall colour and coupling constant factors, are well known:

$$
\begin{gathered}
-i A\left(1_{q}^{-}, 2_{\bar{q}}^{+}, 3_{g}^{-}\right)=-\frac{\langle 13\rangle^{2}}{\langle 12\rangle}, \\
-i A\left(1_{q}^{-}, 2_{\bar{q}}^{+}, 3_{g}^{+}\right)=-\frac{[23]^{2}}{[12]} .
\end{gathered}
$$

B.3 Results for $A\left(1_{q}, 2_{\bar{q}}, 3_{g}, 4_{\bar{l}}, 5_{l}\right)$

The tree-level amplitudes for $q \bar{q} W g$ are also rather simple. Removing the colour and coupling constants as normal we have,

$$
\begin{aligned}
& -i A\left(1_{\bar{q}}^{-}, 2_{\bar{q}}^{+}, 3_{g}^{-}, 4_{\bar{l}}^{+}, 5_{l}^{-}\right)=-\frac{[24]^{2}}{[13][23][45]}, \\
& -i A\left(1_{q}^{-}, 2_{\bar{q}}^{+}, 3_{g}^{+}, 5_{\bar{l}}^{+}, 4_{l}^{-}\right)=\frac{\langle 15\rangle^{2}}{\langle 13\rangle\langle 23\rangle\langle 45\rangle} .
\end{aligned}
$$

\section{Scalar integrals}

We work in the Bjorken-Drell metric so that $l^{2}=l_{0}^{2}-l_{1}^{2}-l_{2}^{2}-l_{3}^{2}$. The definition of the integrals is as follows,

$$
A_{0}\left(m_{1}^{2}\right)=\frac{\mu^{4-D}}{i \pi^{\frac{D}{2}} r_{\Gamma}} \int d^{D} l \frac{1}{\left(l^{2}-m_{1}^{2}\right)},
$$




$$
\begin{aligned}
& B_{0}\left(p_{1} ; m_{1}, m_{2}\right)= \\
& \quad \frac{\mu^{4-D}}{i \pi^{\frac{D}{2}} r_{\Gamma}} \int d^{D} l \frac{1}{\left(l^{2}-m_{1}^{2}\right)\left(\left(l+p_{1}\right)^{2}-m_{2}^{2}\right)}, \\
& C_{0}\left(p_{1}, p_{2} ; m_{1}, m_{2}, m_{3}\right)= \\
& \quad \frac{\mu^{4-D}}{i \pi^{\frac{D}{2}} r_{\Gamma}} \int d^{D} l \frac{1}{\left(l^{2}-m_{1}^{2}\right)\left(\left(l+p_{1}\right)^{2}-m_{2}^{2}\right)\left(\left(l+p_{1}+p_{2}\right)^{2}-m_{3}^{2}\right)}, \\
& D_{0}\left(p_{1}, p_{2}, p_{3} ; m_{1}, m_{2}, m_{3}, m_{4}\right)= \\
& \quad \frac{\mu^{4-D}}{i \pi^{\frac{D}{2}} r_{\Gamma}} \int d^{D} l \frac{1}{\left(l^{2}-m_{1}^{2}\right)\left(\left(l+p_{1}\right)^{2}-m_{2}^{2}\right)\left(\left(l+p_{1}+p_{2}\right)^{2}-m_{3}^{2}\right)\left(\left(l+p_{1}+p_{2}+p_{3}\right)^{2}-m_{4}^{2}\right)} .
\end{aligned}
$$

We have removed the overall constant which occurs in $D$-dimensional integrals

$$
r_{\Gamma} \equiv \frac{\Gamma^{2}(1-\epsilon) \Gamma(1+\epsilon)}{\Gamma(1-2 \epsilon)}=\frac{1}{\Gamma(1-\epsilon)}+\mathcal{O}\left(\epsilon^{3}\right)=1-\epsilon \gamma+\epsilon^{2}\left[\frac{\gamma^{2}}{2}-\frac{\pi^{2}}{12}\right]+\mathcal{O}\left(\epsilon^{3}\right) .
$$

\section{Fermionic self energy}

Introducing the renormalization of the bare parameters, $m_{0}=Z_{m} m$ and $Q_{0}=\sqrt{Z_{Q}} Q$ we may write the renormalized inverse propagator for a heavy quark of momentum $p$ as,

$$
-i \Gamma^{R}(p, m ; g)=Z_{Q}\left[\not p-m-\Sigma(p, m ; g)-m\left(Z_{m}-1\right)\right]+O\left(g^{4}\right) .
$$

$-i \Sigma(p, m ; g)$ is the contribution of the one-loop heavy quark self-energy graph, which prior to renormalization has the form,

$$
\Sigma(p, m ; g)=-g^{2} C_{F} c_{\Gamma}\left[X\left(p^{2}\right)(\not p-m)+m Y\left(p^{2}\right)\right] .
$$

By direct calculation of the Feynman diagram in an arbitrary covariant gauge specified by gauge fixing parameter $\lambda$,

$$
\begin{aligned}
X\left(p^{2}\right)= & {\left[2(1-\delta \epsilon)\left(B_{0}(p ; 0, m)+B_{1}(p ; 0, m)\right)\right.} \\
& \left.-(1-\lambda)\left(B_{0}(p ; 0, m)+\left(p^{2}-m^{2}\right) B_{0}^{\prime}(p ; 0, m)\right)\right] \\
Y\left(p^{2}\right)= & {\left.\left[2 B_{1}(p, 0, m)(1-\delta \epsilon)-2 B_{0}(p ; 0, m)-(1-\lambda)\left(p^{2}-m^{2}\right) B_{0}^{\prime}(p ; 0, m)\right)\right] }
\end{aligned}
$$

where $\delta=0$ in the FDH scheme, $\delta=1$ in the conventional dimensional regularization scheme, and $C_{F}=\frac{N_{c}^{2}-1}{2 N_{c}}$. The integrals $B_{0}$ and $B_{1}$ are defined as,

$$
\left\{B_{0}(p ; 0, m), B_{1}(p ; 0, m) p^{\mu}\right\}=\frac{\mu^{4-D}}{i \pi^{\frac{D}{2}} r_{\Gamma}} \int d^{D} l \frac{\left\{1, l^{\mu}\right\}}{l^{2}\left((l+p)^{2}-m^{2}\right)},
$$


and $B_{i}^{\prime}$ is the derivative of the form factor $B_{i}$ with respect to $p^{2}$.

Taking the limit $p^{2}=m^{2}$ before the limit $\epsilon \rightarrow 0$, we have the following results,

$$
\begin{aligned}
& \left.B_{0}(p ; 0, m)\right|_{p^{2}=m^{2}}=\left(\frac{\mu^{2}}{m^{2}}\right)^{\epsilon}\left[\frac{1}{\epsilon}+2\right], \\
& \left.B_{1}(p ; 0, m)\right|_{p^{2}=m^{2}}=\left(\frac{\mu^{2}}{m^{2}}\right)^{\epsilon}\left[-\frac{1}{2 \epsilon}-\frac{1}{2}\right], \\
& \left.B_{0}^{\prime}(p ; 0, m)\right|_{p^{2}=m^{2}}=-\frac{1}{2 m^{2}}\left(\frac{\mu^{2}}{m^{2}}\right)^{\epsilon}\left[\frac{1}{\epsilon}+2\right], \\
& \left.B_{1}^{\prime}(p ; 0, m)\right|_{p^{2}=m^{2}}=-\frac{1}{2 m^{2}} .
\end{aligned}
$$

The mass renormalization is fixed by the condition that the inverse propagator vanish on shell,

$$
Z_{m}=1+g^{2} C_{F} Y\left(m^{2}\right)=1-c_{\Gamma} g^{2} C_{F}\left[\frac{3}{\epsilon}+3 \ln \left(\frac{\mu^{2}}{m^{2}}\right)+5-\delta\right]+O\left(g^{4}, \epsilon\right) .
$$

After mass renormalization the result for the inverse propagator becomes,

$$
-i \Gamma^{R}(p, m ; g)=Z_{Q}\left[(\not p-m)\left(1+g^{2} C_{F} c_{\Gamma} X\left(p^{2}\right)\right)+m C_{F} c_{\Gamma}\left(Y\left(p^{2}\right)-Y\left(m^{2}\right)\right)\right] .
$$

Renormalizing the wave function at the point $p^{2}=m^{2}$ we find,

$$
Z_{Q}=1-g^{2} C_{F} c_{\Gamma}\left[X\left(m^{2}\right)+\left.2 m^{2} \frac{d Y\left(p^{2}\right)}{d p^{2}}\right|_{p^{2}=m^{2}}\right]+O\left(g^{4}\right) .
$$

By explicit calculation we have that,

$$
\begin{aligned}
X\left(m^{2}\right) & =\left[\frac{1}{\epsilon}+\ln \left(\frac{\mu^{2}}{m^{2}}\right)+(3-\delta)-(1-\lambda)\left(\frac{1}{\epsilon}+\ln \left(\frac{\mu^{2}}{m^{2}}\right)+2\right)+O(\epsilon)\right], \\
\left.2 m^{2} \frac{d Y\left(p^{2}\right)}{d p^{2}}\right|_{p^{2}=m^{2}} & =\left[\frac{2}{\epsilon}+2 \ln \left(\frac{\mu^{2}}{m^{2}}\right)+2+(1-\lambda)\left(\frac{1}{\epsilon}+\ln \left(\frac{\mu^{2}}{m^{2}}\right)+2\right)+O(\epsilon)\right] .
\end{aligned}
$$

The final result for the wave function renormalization is independent of the gauge fixing parameter, $\lambda$,

$$
Z_{Q}=1-g^{2} c_{\Gamma} C_{F}\left[\frac{3}{\epsilon}+3 \ln \left(\frac{\mu^{2}}{m^{2}}\right)+5-\delta\right]+O\left(g^{4}, \epsilon\right) .
$$

The agreement between eq. (D.6) and eq. (D.10) is fortuitous because the former contains only ultraviolet poles, whereas the latter contains both ultraviolet and infrared poles.

\section{E Numerical evaluation}

For the convenience of the reader we present numerical results for the amplitudes at a particular phase space point. The results we present contain no ultraviolet renormalization and the self energy corrections on the two massive external legs have not been included. (The self energy corrections on the massless external legs vanish). 


\begin{tabular}{|l|c|c|c|c|c|c|}
\hline & \multicolumn{3}{|c|}{$A_{6}\left(1_{q}^{-}, 2_{\bar{Q}}^{+}, 3_{Q}^{-}, 4_{\bar{q}}^{+}, 5_{\bar{\ell}}^{+}, 6_{\ell}^{-}\right)$} & \multicolumn{3}{c|}{$A_{6}\left(1_{q}^{-}, 2_{\bar{Q}}^{-}, 3_{Q}^{+}, 4_{\bar{q}}^{+}, 5_{\bar{\ell}}^{+}, 6_{\ell}^{-}\right)$} \\
\hline & $1 / \epsilon^{2}$ & $1 / \epsilon$ & $\epsilon^{0}$ & $1 / \epsilon^{2}$ & $1 / \epsilon$ & $\epsilon^{0}$ \\
\hline$-i A_{6}^{\text {tree }}$ & 0 & 0 & -0.02873880764 & 0 & 0 & 0.00703966555 \\
& & & $0.00020478685 i$ & & & $0.08491754257 i$ \\
\hline$A_{6}^{\text {Ic }} / A_{6}^{\text {tree }}$ & -1 & 3.84296991603 & 21.70930260416 & -1 & 3.84296991603 & 21.65220761331 \\
& & & $6.25248592358 i$ & & & $2.82472065470 i$ \\
\hline$A_{6}^{\text {cb }} / A_{6}^{\text {tree }}$ & 1 & -3.27408593656 & -20.64278559885 & 1 & -3.27408593656 & -20.63395981655 \\
& & & $-3.14315195865 i$ & & & $-4.57661106366 i$ \\
\hline$A_{6}^{\text {sl }} / A_{6}^{\text {tree }}$ & -1 & 0.72362882832 & 22.03005675285 & -1 & 0.72362882832 & 21.99641271870 \\
& & $-7.83563162387 i$ & $-22.49326728353 i$ & & $-7.83563162387 i$ & $-22.71671278884 i$ \\
\hline$A_{6}^{\text {If }} / A_{6}^{\text {tree }}$ & 0 & 0.66666666667 & 2.12677656298 & 0 & 0.666666666667 & 2.12677656298 \\
& & & $2.09439510239 i$ & & & $2.09439510239 i$ \\
\hline
\end{tabular}

Table 6. Numerical values of primitive amplitudes at the kinematic point defined in eqs. (2.5), (E.1).

The phase space point is specified by six momenta satisfying overall momentum conservation $k_{1}+p_{2}+p_{3}+k_{4}+k_{5}+k_{6}=0$ and $k_{i}^{2}=0, p_{2}^{2}=p_{3}^{2}=m^{2}$. The massive momenta $p_{2}$ and $p_{3}$ are defined in eqs. (2.5), (2.6). For the numerical results the massless momenta $k_{i}$ are taken to be,

$$
\begin{aligned}
& k_{1}=\frac{\mu}{2}(-1,+\sin \theta,+\cos \theta \sin \phi,+\cos \theta \cos \phi), \\
& k_{2}=\frac{\mu}{3}(1,1,0,0), \\
& k_{3}=\frac{\mu}{7}(1, \cos \sigma, \sin \sigma, 0), \\
& k_{4}=\frac{\mu}{2}(-1,-\sin \theta,-\cos \theta \sin \phi,-\cos \theta \cos \phi), \\
& k_{5}=\frac{\mu}{6}(1, \cos \rho \cos \sigma, \cos \rho \sin \sigma, \sin \rho), \\
& k_{6}=-k_{1}-k_{2}-k_{3}-k_{4}-k_{5},
\end{aligned}
$$

where $\theta=\pi / 4, \phi=\pi / 6, \rho=\pi / 3, \cos \sigma=-7 / 19, m=1 \mathrm{GeV}$ and $\mu=6 \mathrm{GeV}$. Note that the energies of $k_{1}$ and $k_{4}$ are negative and $k_{i}^{2}=0$. As usual $\mu$ also denotes the scale which is used to carry the dimensionality of the $D$-dimensional integrals. With $m=1 \mathrm{GeV}$ we have $\beta \sim 0.38397382 \ldots$ so that $\beta$ differs substantially from the massless limit, $\beta=1$.

The results for the primitive amplitudes are presented in tables 6 and 7, where we have divided the 1-loop amplitudes by their tree-level counterparts in order to remove the overall ambiguity in the phase. ${ }^{1}$ The individual amplitudes labelled by the particular spin labels are dependent on our choice of the spinor wave functions, eq. (2.9). Results independent of this convention may be obtained by summing the squares of the four amplitudes. There is enough information in tables 6 and 7 to reconstruct the one-loop amplitude $A_{6 ; 1}$ using eq. (3.13) and hence to reconstruct the spin-summed interference with the lowest order amplitude.

The results in tables 6 and 7 have been checked by an independent program.

\footnotetext{
${ }^{1}$ Our phase for the spinor products can be understood from the routine spinoru.f in the MCFM distribution.
} 


\begin{tabular}{|c|c|c|c|c|c|c|}
\hline & \multicolumn{3}{|c|}{$A_{6}\left(1_{q}^{-}, 2_{\bar{Q}}^{-}, 3_{Q}^{-}, 4_{\bar{q}}^{+}, 5_{\bar{\ell}}^{+}, 6_{\ell}^{-}\right)$} & \multicolumn{3}{c|}{$A_{6}\left(1_{q}^{-}, 2_{\bar{Q}}^{+}, 3_{Q}^{+}, 4_{\bar{q}}^{+}, 5_{\bar{\ell}}^{+}, 6_{\ell}^{-}\right)$} \\
\hline & $1 / \epsilon^{2}$ & $1 / \epsilon$ & $\epsilon^{0}$ & $1 / \epsilon^{2}$ & $1 / \epsilon$ & $\epsilon^{0}$ \\
\hline$-i A_{6}^{\text {tree }}$ & 0 & 0 & -0.03716837450 & 0 & 0 & -0.03716837450 \\
& & & $0.03263223728 i$ & & & $0.03263223728 i$ \\
\hline$A_{6}^{\text {lc }} / A_{6}^{\text {tree }}$ & -1 & 3.84296991603 & 16.95522192600 & -1 & 3.84296991603 & 24.66977363941 \\
& & & $5.96305954078 i$ & & & $4.27788837815 i$ \\
\hline$A_{6}^{\text {cb }} / A_{6}^{\text {tree }}$ & 1 & -3.27408593656 & -25.16188693978 & 1 & -3.27408593656 & -17.34322884729 \\
& & & $-4.09626473381 i$ & & & $-4.76582819889 i$ \\
\hline$A_{6}^{\text {sl }} / A_{6}^{\text {tree }}$ & -1 & 0.72362882832 & 21.88454128871 & -1 & 0.72362882832 & 21.88454128871 \\
& & $-7.83563162387 i$ & $-21.57825725817 i$ & & $-7.83563162387 i$ & $-21.57825725817 i$ \\
\hline$A_{6}^{\text {If }} / A_{6}^{\text {tree }}$ & 0 & 0.66666666667 & 2.12677656298 & 0 & 0.666666666667 & 2.12677656298 \\
& & & $2.09439510239 i$ & & & $2.09439510239 i$ \\
\hline
\end{tabular}

Table 7. Numerical values of primitive amplitudes at the kinematic point defined in eqs. (2.5), (E.1).

Open Access. This article is distributed under the terms of the Creative Commons Attribution Noncommercial License which permits any noncommercial use, distribution, and reproduction in any medium, provided the original author(s) and source are credited.

\section{References}

[1] R.K. Ellis and S. Veseli, Strong radiative corrections to Wbb production in $p \bar{p}$ collisions, Phys. Rev. D 60 (1999) 011501 [hep-ph/9810489] [SPIRES].

[2] F. Febres Cordero, L. Reina and D. Wackeroth, NLO QCD corrections to W boson production with a massive b-quark jet pair at the Tevatron $p \bar{p}$ collider, Phys. Rev. D 74 (2006) 034007 [hep-ph/0606102] [SPIRES].

[3] F. Febres Cordero, L. Reina and D. Wackeroth, $W$ - and Z-boson production with a massive bottom-quark pair at the Large Hadron Collider, Phys. Rev. D 80 (2009) 034015 [arXiv:0906.1923] [SPIRES].

[4] F.F. Cordero, L. Reina and D. Wackeroth, Associated production of a $W$ or $Z$ boson with bottom quarks at the Tevatron and the LHC, PoS RADCOR2009 (2010) 055 [arXiv: 1001.3362] [SPIRES].

[5] J.M. Campbell et al., Associated production of a $W$ boson and one $b$ jet, Phys. Rev. D 79 (2009) 034023 [arXiv: 0809.3003] [SPIRES].

[6] CDF AND D0 collaboration, Combined CDF and D0 upper limits on standard model Higgs-boson production with up to $6.7 \mathrm{fb}^{-1}$ of data, arXiv:1007.4587 [SPIRES].

[7] J.M. Butterworth, A.R. Davison, M. Rubin and G.P. Salam, Jet substructure as a new Higgs search channel at the LHC, Phys. Rev. Lett. 100 (2008) 242001 [arXiv:0802.2470] [SPIRES].

[8] Z. Bern, L.J. Dixon, D.C. Dunbar and D.A. Kosower, One-loop n-point gauge theory amplitudes, unitarity and collinear limits, Nucl. Phys. B 425 (1994) 217 [hep-ph/9403226] [SPIRES].

[9] Z. Bern, L.J. Dixon, D.C. Dunbar and D.A. Kosower, Fusing gauge theory tree amplitudes into loop amplitudes, Nucl. Phys. B 435 (1995) 59 [hep-ph/9409265] [SPIRES]. 
[10] R. Britto, F. Cachazo and B. Feng, Generalized unitarity and one-loop amplitudes in $N=4$ super-Yang-Mills, Nucl. Phys. B 725 (2005) 275 [hep-th/0412103] [SPIRES].

[11] R. Britto, E. Buchbinder, F. Cachazo and B. Feng, One-loop amplitudes of gluons in SQCD, Phys. Rev. D 72 (2005) 065012 [hep-ph/0503132] [SPIRES].

[12] R. Britto, B. Feng and P. Mastrolia, The cut-constructible part of QCD amplitudes, Phys. Rev. D 73 (2006) 105004 [hep-ph/0602178] [SPIRES].

[13] D. Forde, Direct extraction of one-loop integral coefficients, Phys. Rev. D 75 (2007) 125019 [arXiv:0704.1835] [SPIRES].

[14] R. Kleiss and W.J. Stirling, Spinor techniques for calculating $p \bar{p} \rightarrow W^{ \pm} / Z_{0}+$ Jets, Nucl. Phys. B 262 (1985) 235 [SPIRES].

[15] S. Badger, Unitarity methods for one-loop amplitudes, PoS (RADCOR2009) 020 [SPIRES].

[16] S. Badger, R. Sattler and V. Yundin, Analytic computations of massive one-loop amplitudes, Nucl. Phys. Proc. Suppl. 205-206 (2010) 61 [arXiv:1006.5011] [SPIRES].

[17] G. Rodrigo, Multigluonic scattering amplitudes of heavy quarks, JHEP 09 (2005) 079 [hep-ph/0508138] [SPIRES].

[18] Z. Bern, L.J. Dixon and D.A. Kosower, One-loop amplitudes for $e^{+} e^{-}$to four partons, Nucl. Phys. B 513 (1998) 3 [hep-ph/9708239] [SPIRES].

[19] R.K. Ellis and G. Zanderighi, Scalar one-loop integrals for QCD, JHEP 02 (2008) 002 [arXiv:0712.1851] [SPIRES].

[20] F. Cachazo, P. Svrček and E. Witten, MHV vertices and tree amplitudes in gauge theory, JHEP 09 (2004) 006 [hep-th/0403047] [SPIRES].

[21] Z. Bern, A. De Freitas, L.J. Dixon and H.L. Wong, Supersymmetric regularization, two-loop QCD amplitudes and coupling shifts, Phys. Rev. D 66 (2002) 085002 [hep-ph/0202271] [SPIRES].

[22] J.A.M. Vermaseren, New features of FORM, math-ph/0010025 [SPIRES].

[23] S. Catani, S. Dittmaier and Z. Trócsányi, One-loop singular behaviour of QCD and SUSY QCD amplitudes with massive partons, Phys. Lett. B 500 (2001) 149 [hep-ph/0011222] [SPIRES].

[24] Z. Bern, L.J. Dixon and D.A. Kosower, Dimensionally regulated pentagon integrals, Nucl. Phys. B 412 (1994) 751 [hep-ph/9306240] [SPIRES].

[25] S. Badger, J.M. Campbell, R.K. Ellis and C. Williams, Analytic results for the one-loop NMHV Hqqgg amplitude, JHEP 12 (2009) 035 [arXiv: 0910.4481] [SPIRES].

[26] G. Passarino and M.J.G. Veltman, One loop corrections for $e^{+} e^{-}$annihilation into $\mu^{+} \mu^{-}$in the Weinberg model, Nucl. Phys. B 160 (1979) 151 [SPIRES].

[27] L.J. Dixon and Y. Sofianatos, Analytic one-loop amplitudes for a Higgs boson plus four partons, JHEP 08 (2009) 058 [arXiv:0906.0008] [SPIRES].

[28] Z. Kunszt, A. Signer and Z. Trócsányi, One loop helicity amplitudes for all $2 \rightarrow 2$ processes in QCD and $N=1$ supersymmetric Yang-Mills theory, Nucl. Phys. B 411 (1994) 397 [hep-ph/9305239] [SPIRES].

[29] S.D. Badger, Generalised unitarity at one-loop with massive fermions, Nucl. Phys. Proc. Suppl. 183 (2008) 220 [arXiv:0807.1245] [SPIRES].

[30] J.C. Collins, F. Wilczek and A. Zee, Low-energy manifestations of heavy particles: application to the neutral current, Phys. Rev. D 18 (1978) 242 [SPIRES]. 
[31] J.M. Campbell and R.K. Ellis, MCFM home page, http://mcfm.fnal.gov.

[32] J.M. Campbell and R.K. Ellis, An update on vector boson pair production at hadron colliders, Phys. Rev. D 60 (1999) 113006 [hep-ph/9905386] [SPIRES].

[33] R.K. Ellis, D.A. Ross and A.E. Terrano, The Perturbative Calculation of Jet Structure in $e^{+} e^{-}$Annihilation, Nucl. Phys. B 178 (1981) 421 [SPIRES].

[34] S. Catani and M.H. Seymour, A general algorithm for calculating jet cross sections in NLO QCD, Nucl. Phys. B 485 (1997) 291 [Erratum ibid. B 510 (1998) 503] [hep-ph/9605323] [SPIRES].

[35] S. Catani, S. Dittmaier, M.H. Seymour and Z. Trócsányi, The dipole formalism for next-to-leading order QCD calculations with massive partons, Nucl. Phys. B 627 (2002) 189 [hep-ph/0201036] [SPIRES].

[36] R.K. Ellis, W.T. Giele, Z. Kunszt, K. Melnikov and G. Zanderighi, One-loop amplitudes for $W^{+} 3$ jet production in hadron collisions, JHEP 01 (2009) 012 [arXiv:0810.2762] [SPIRES].

[37] Z. Nagy and Z. Trócsányi, Next-to-leading order calculation of four-jet observables in electron positron annihilation, Phys. Rev. D 59 (1999) 014020 [Erratum ibid. D 62 (2000) 099902] [hep-ph/9806317] [SPIRES].

[38] Z. Nagy, Next-to-leading order calculation of three jet observables in hadron hadron collision, Phys. Rev. D 68 (2003) 094002 [hep-ph/0307268] [SPIRES].

[39] J.M. Campbell, R.K. Ellis and D.L. Rainwater, Next-to-leading order QCD predictions for $W+2$ jet and $Z+2$ jet production at the CERN LHC, Phys. Rev. D 68 (2003) 094021 [hep-ph/0308195] [SPIRES].

[40] A.G. Morgan, Second order fermions in gauge theories, Phys. Lett. B 351 (1995) 249 [hep-ph/9502230] [SPIRES]. 